In Situ

Revue des patrimoines
In Situ

Revue des patrimoines

29 | 2016

Ensembles mobiliers, industriels, techniques.

Connaissance, protection, conservation, présentation au public

\title{
Les scieries hydrauliques vosgiennes
}

The hydraulic saw-mills in the Vosges department

\section{Jean-Yves Henry}

\section{(2) OpenEdition}

\section{Journals}

Édition électronique

URL : http://journals.openedition.org/insitu/12965

DOI : 10.4000/insitu. 12965

ISSN : 1630-7305

\section{Éditeur}

Ministère de la culture

Référence électronique

Jean-Yves Henry, "Les scieries hydrauliques vosgiennes », In Situ [En ligne], 29 | 2016, mis en ligne le 13 juillet 2016, consulté le 30 avril 2019. URL : http://journals.openedition.org/insitu/12965 ; DOI :

10.4000/insitu. 12965

Ce document a été généré automatiquement le 30 avril 2019.

\section{c) (i) $९$}

In Situ Revues des patrimoines est mis à disposition selon les termes de la licence Creative Commons Attribution - Pas d'Utilisation Commerciale - Pas de Modification 4.0 International. 


\section{Les scieries hydrauliques vosgiennes}

The hydraulic saw-mills in the Vosges department

Jean-Yves Henry

\section{Le cadre de l'étude d'inventaire}

1 L'étude thématique de la petite industrie hydraulique a été conduite entre 2010 et 2012 par l'Inventaire général de la région Lorraine dans le cadre d'une opération plus générale consacrée à l'architecture rurale de la montagne vosgienne. Le choix de cette thématique avait pour objectif de mieux comprendre la pluriactivité en milieu agricole et mieux cerner les liens entre agriculture et petite industrie. En effet, les activités artisanales, voire commerciales, permettent souvent aux agriculteurs-éleveurs de compléter leurs revenus et elles sont intimement mêlées. On rencontre des activités préindustrielles dans les fermes et des activités agro-pastorales dans les petits édifices industriels (fig. 1). Ces sites, jusqu'au milieu du XIX ${ }^{\mathrm{e}}$ siècle, utilisent exclusivement l'énergie hydraulique des nombreux cours d'eau du massif, même à très faible débit. Les moulins «à sang " y sont quasiment inconnus, l'emploi de la traction animale étant réservé aux travaux des champs ou de la forêt. L'étude visait aussi à comprendre l'évolution des sites : succession des industries sur un même lieu, évolution des techniques, industrialisation des filières, place de cette petite industrie dans le développement économique de la montagne vosgienne ainsi que son rôle dans l'évolution paysagère des Hautes-Vosges. 
Figure 1

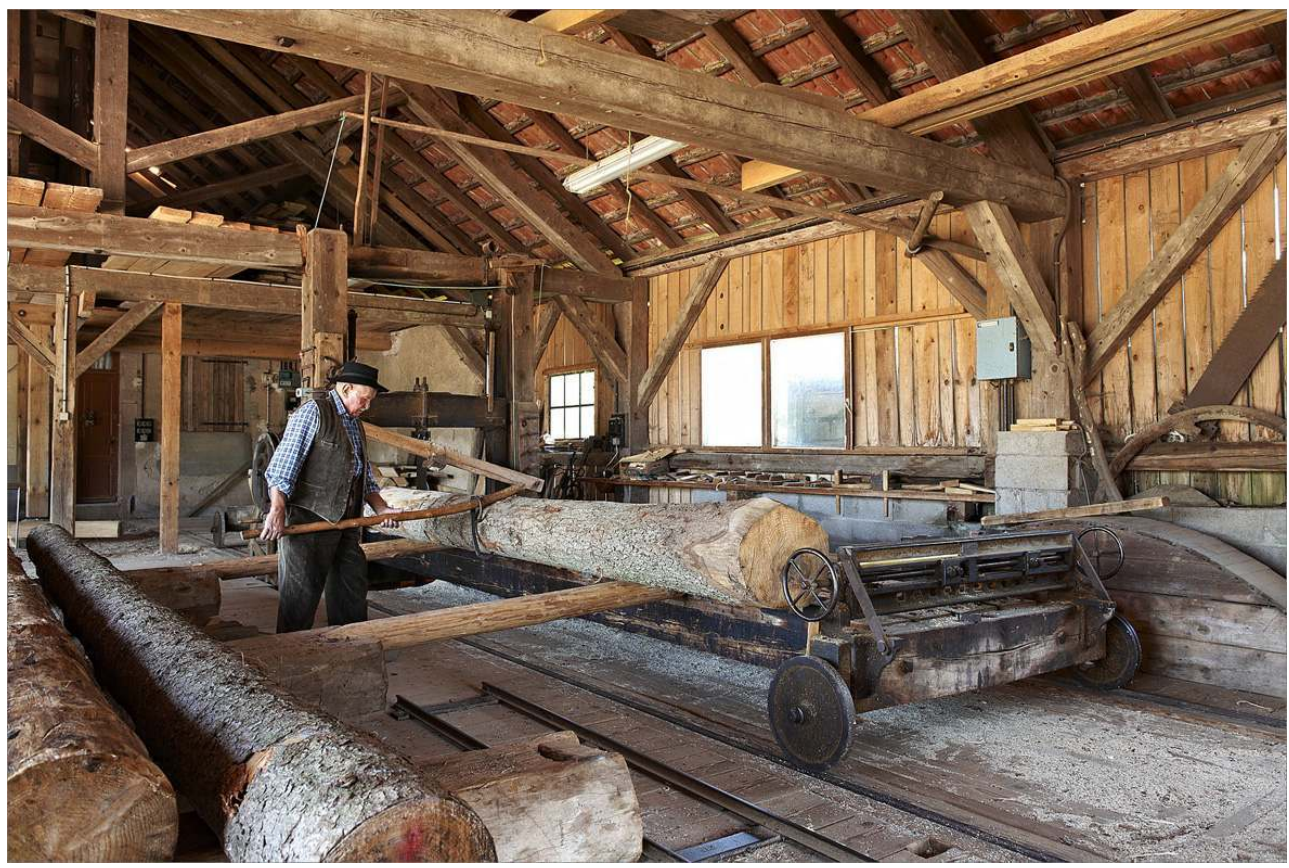

Sagard en train de charger une grume sur le chariot de la scie, scierie de Mandray (site F, voir tableau en annexe).

Phot. Drapier, Bertrand. (Base Mémoire : IVR41_20108810734NUC2A). ( Région Lorraine-Inventaire général.

2 La petite industrie hydraulique étudiée se compose de moulins à céréales - chaque village en comptait au moins un - de scieries, de petits ateliers de transformation du bois (saboterie, tournerie, boissellerie, fabrication de boîtes à fromage, menuiserie) complétés à partir du XIX ${ }^{\mathrm{e}}$ siècle par les graniteries ${ }^{1}$, féculeries et les petits ateliers textiles (filatures et tissages) (fig. 2, fig. 3). 
Figure 2

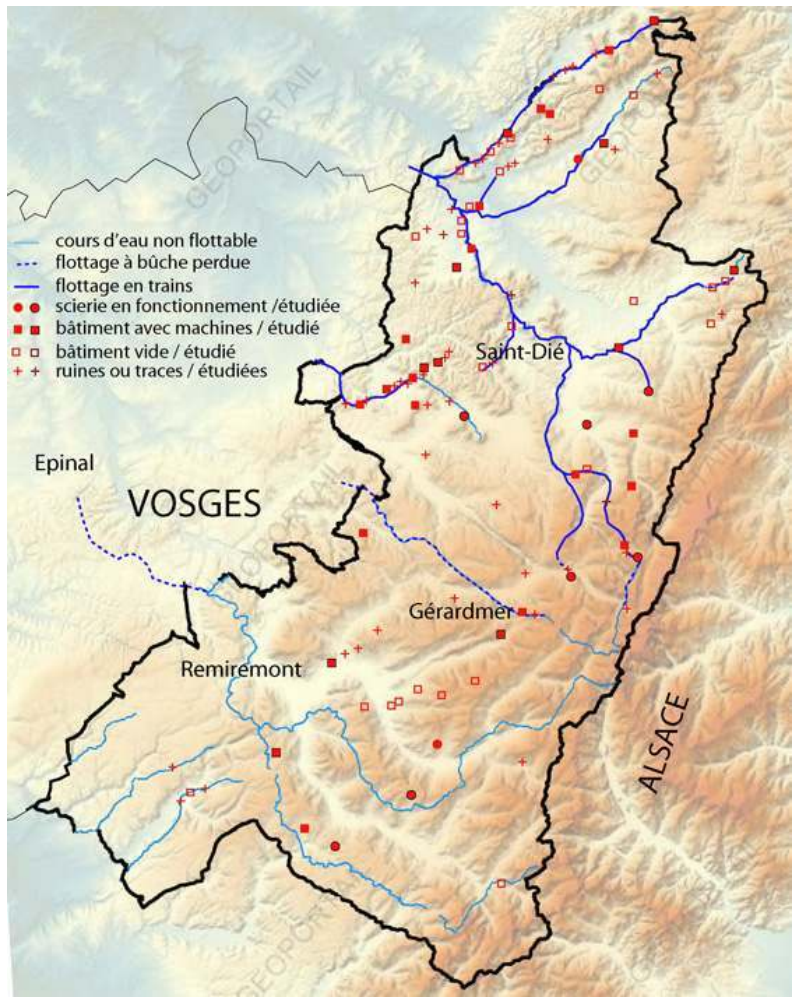

Cartographie des scieries repérées ou étudiées.

Cart. Henry, Jean-Yves. (Base Mémoire : IVR41_20128812190NUDA). @ Région Lorraine-Inventaire général.

Figure 3

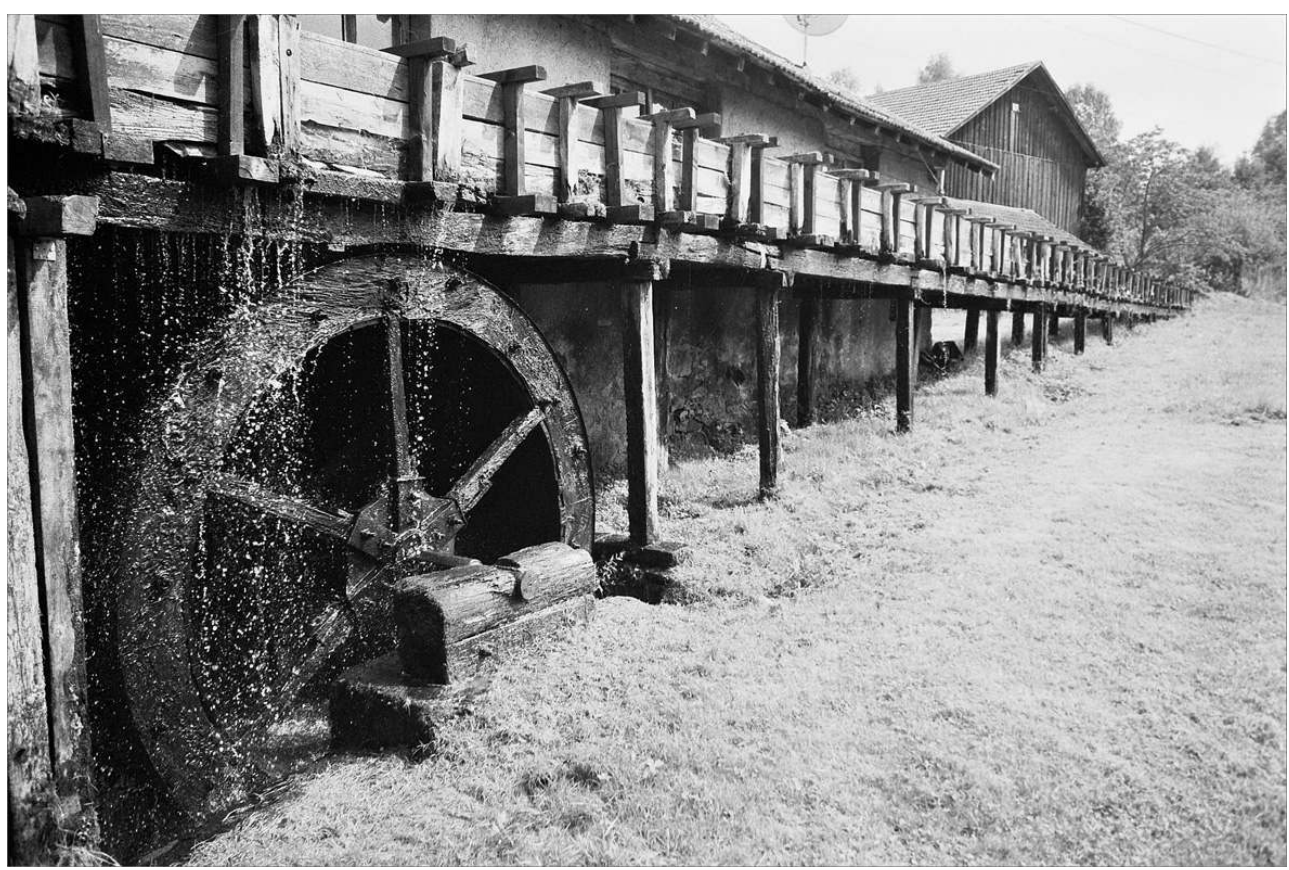

Canal d'amenée d'eau, scierie de Ban-de-Laveline (site A)

Phot. Grandidier, Daniel. (Base Mémoire : IVR41_938800576NUC2). @ Région Lorraine-Inventaire général. 
Dans cet ensemble, les scieries occupent une place prépondérante, justifiée à la fois par l'importance de cette industrie dans la région fortement forestière et par la bonne conservation de ces édifices. Contrairement aux moulins situés au cœur des hameaux et aujourd'hui quasiment tous disparus, les scieries ont mieux résisté à la pression immobilière en raison de leur localisation à la périphérie des bourgs, à proximité de la forêt et le long d'un cours d'eau trop petit pour nécessiter le renouvellement de son exploitation au cours $\mathrm{du} \mathrm{xx}^{\mathrm{e}}$ siècle. Leur prédominance nous a conduits à les étudier spécifiquement : 115 sites ont ainsi été repérés sur le territoire de la montagne vosgienne. Leur état de conservation est tout à fait variable : 43 sont dénaturés ou en ruines, mais 33 conservent encore leurs machines parmi lesquels 10 sont encore en état de fonctionnement et 7 ouverts au public.

4 L'étude ne porte pas tant sur les scieries, "usines où l'on scie le bois ", de peu d'intérêt architectural, que sur la machinerie qu'elles abritent et sur les savoir-faire liés. La machinerie est composée d'un moteur hydraulique (roue ou turbine), d'un système de transmission de puissance (engrenages et/ou courroie) et de la scie, l'ensemble étant immeuble par destination. Le bâtiment se compose d'un sous-sol maçonné destiné à supporter la machinerie et à la protéger du cours d'eau voisin, surmonté généralement d'un hangar en bois abritant la halle de sciage des intempéries et d'un petit logement accolé. L'étude des scieries porte donc avant tout sur les machines, mais ces dernières sont indissociables de leur environnement: cours d'eau, canal d'amenée, canal de décharge, réservoir et topographie du site; ce sont les caractéristiques de ces éléments (débit et hauteur de chute) qui vont déterminer le type de moteur hydraulique employé et la capacité de la machine qui l'accompagne.

Les scieries vosgiennes qui nous sont parvenues ont toutes fait l'objet de transformations majeures dans la seconde moitié $d u x^{e}{ }^{e}$ siècle ou au début $d u x^{e}$ siècle, tant sur la scie elle-même que sur son moteur hydraulique. Ces transformations destinées à améliorer les performances des machines sont concomitantes à l'apparition d'une importante industrie métallurgique (fonderie et atelier de mécanique) à proximité. Les documents qui auraient pu nous permettre de comprendre les origines de ces mécanismes sont extrêmement rares, ces industries ayant quasiment toutes disparu sans laisser d'archives.

\section{Historique des techniques de sciage}

\section{Du moulin à scier au haut-fer}

6 Le « moulin à scier », terme généralement employé dans les archives médiévales, est une machine à lame verticale animée d'un mouvement alternatif (connue dans la littérature sous le nom de scie à cadre) basée sur la technique du sciage de long et entraînée par une roue hydraulique. Elle est connue dans ses principes depuis le haut Moyen Âge et sa construction est attestée dans toute l'Europe et bien étudiée autour de l'arc alpin. 


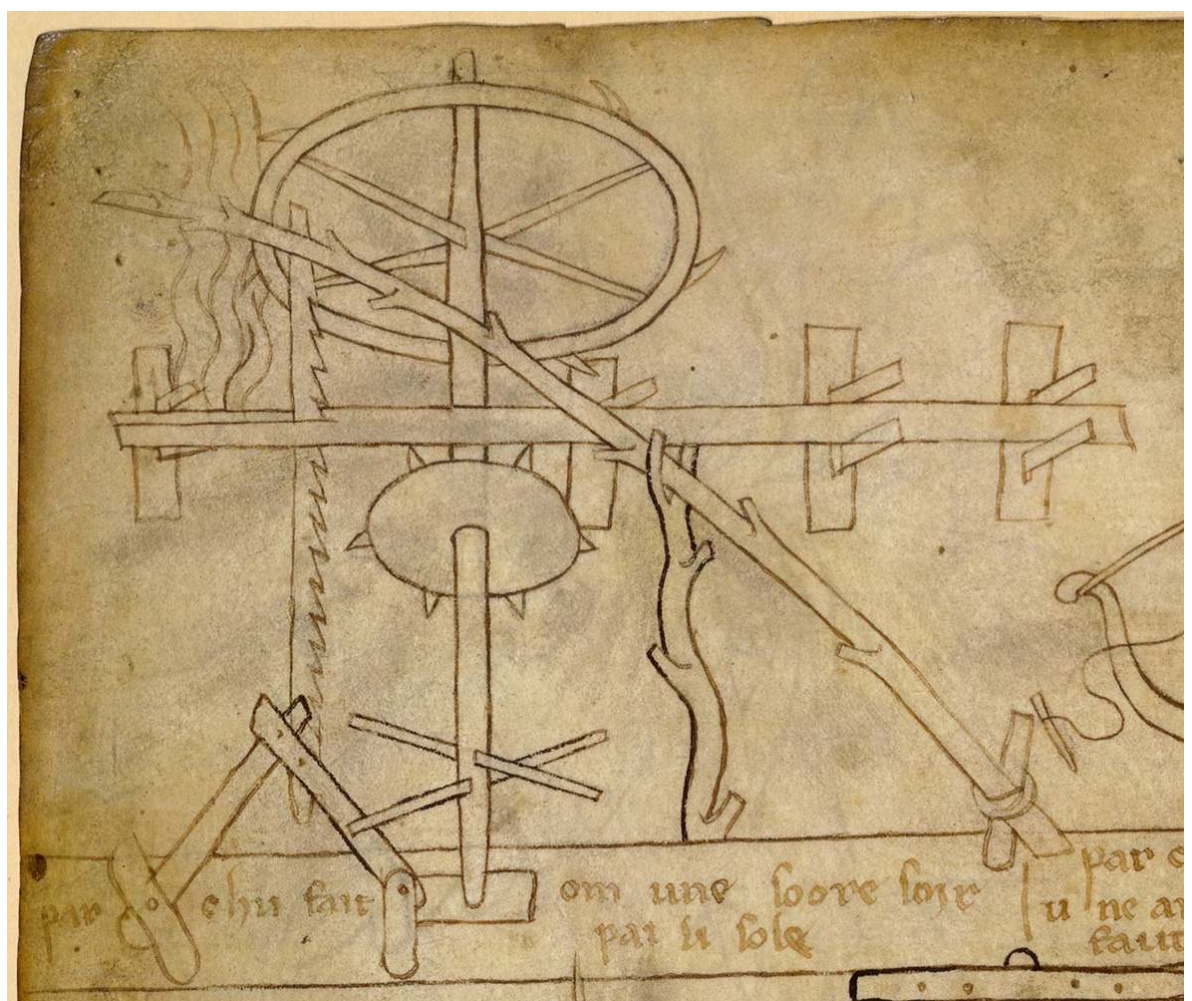

Premier dessin d'une scie hydraulique. Partie d'un dessin à la plume de Villard de Honnecourt, vers 1230. Sources Bibliothèque nationale de France, SNR3, $f^{\circ}$ 44. (Base Mémoire: IVR41_20118810013NUC2A)

(c) Bibliothèque nationale de France. @ C Région Lorraine-Inventaire général.

7 Villard de Honnecourt en donne les principes dans un dessin vers 1230 (fig. 4). Ce plan schématique, même s'il reste difficile à interpréter, montre les principes à mettre en œuvre : transformer le mouvement de rotation de la roue en mouvement de translation alternatif de la lame, guider la pièce de bois à scier et la faire avancer dans un mouvement lié à celui de la lame. Ces trois principes, mis en œuvre par des solutions technologiques diverses dans l'espace et le temps, resteront intangibles jusqu'au début du $\mathrm{xx}^{\mathrm{e}}$ siècle où la scie à ruban finira par les détrôner. 


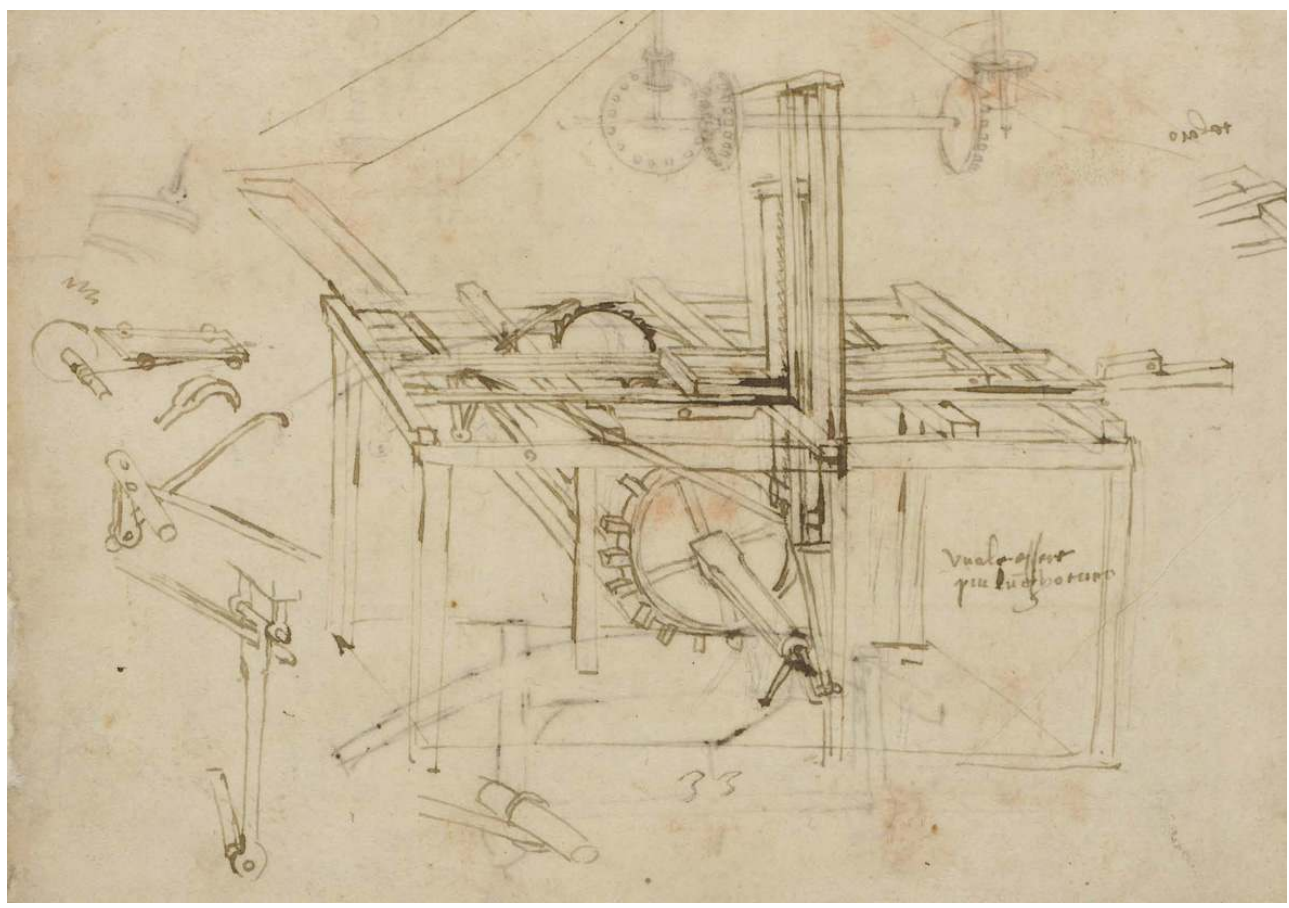

Dessin d'une scie hydraulique. Partie d'un dessin à la plume de Léonard de Vinci vers 1480. Sources bibliothèque Ambrosienne, Milan, Codex atlantica f॰ 389. (Base Mémoire : IVR41_20118810014NUC2A).

(c) Bibliothèque Ambrosienne, Milan. (c) Région Lorraine-Inventaire général.

8 Léonard de Vinci en donne vers 1480 un dessin surprenant : la lame guidée par un cadre est entraînée par l'intermédiaire d'un système bielle-manivelle par une roue hydraulique par le dessous, le mouvement du chariot est synchronisé à celui de la lame par une perche et une roue à rochet $^{2}$ (fig. 5). Or, cette évolution de la scie à cadre caractérisée par l'emploi du système bielle-manivelle ne trouve sa première attestation, du moins dans les Vosges, qu'en $1836^{3}$ et ne sera généralisée qu'à la fin du XIX ${ }^{\mathrm{e}}$ siècle, ce type de scie recevant alors l'appellation « haut-fer » ou « scie à manivelle ».

Auparavant, les sources font référence à des "scies à bloc ", plus localement appelées " scies à plomb ", caractérisées par l'emploi d'un arbre à excentrique pour transmettre le mouvement de la roue au cadre qui porte la lame. Ce dernier est propulsé vers le haut par le choc de l'excentrique sur le cadre, qui en retombant de toute sa masse, scie.

Les archives ${ }^{4}$ montrent que toutes les scieries domaniales de la vallée de la Plaine sont dites « à plomb ou à came » lors de l'inventaire de 1852 et que lors de celui de 1912, elles sont toutes dotées au minimum d'un haut-fer, voire d'une seconde scie. Dès 1860 , la transformation systématique commence, elle nécessite souvent la reconstruction totale de l'édifice. De plus, elle est généralement accompagnée, pour les scieries situées sur de petits cours d'eau, par le remplacement de la roue hydraulique par une turbine. Cette dernière modification entraîne le creusement d'un étang de retenue d'eau, l'installation d'une conduite forcée et donc le réaménagement des biefs.

11 Les machines conservées sur le territoire de l'étude forment un ensemble homogène du point de vue du mécanisme de la scie: scie à manivelle avec un chariot guidé par des roues et entraîné par friction par un rouleau, fixation de la grume sur le chariot par 
griffes ou pinces. Ceci les distingue fortement des scieries de l'Europe alpine étudiées par Herbert Jüttemann dans Alte Bauernsägen ${ }^{5}$ qui reconnaît des scieries de type augsbourgeois (type A dont l'épicentre serait la ville d'Augsbourg, en Bavière) au nord de l'arc alpin, et de type vénitien (type B) au sud. Scieries dont le type chariot et son entraînement sont fort différents des scieries vosgiennes qui nous sont parvenues.

L'absence d'une étude générale des scieries sur l'ensemble du territoire français et la pauvreté des plans de scie à bloc, précis et localisés, ne nous permettent pas de rattacher formellement les scieries vosgiennes et plus généralement, celles du nord-est de l'Europe, au type A, même si les techniques de construction de quelques scieries belges de la région de Virton (scierie Naisse à Virton et moulin Clément à Saint-Léger) semblent s'en rapprocher (fig. 6).

Figure 6

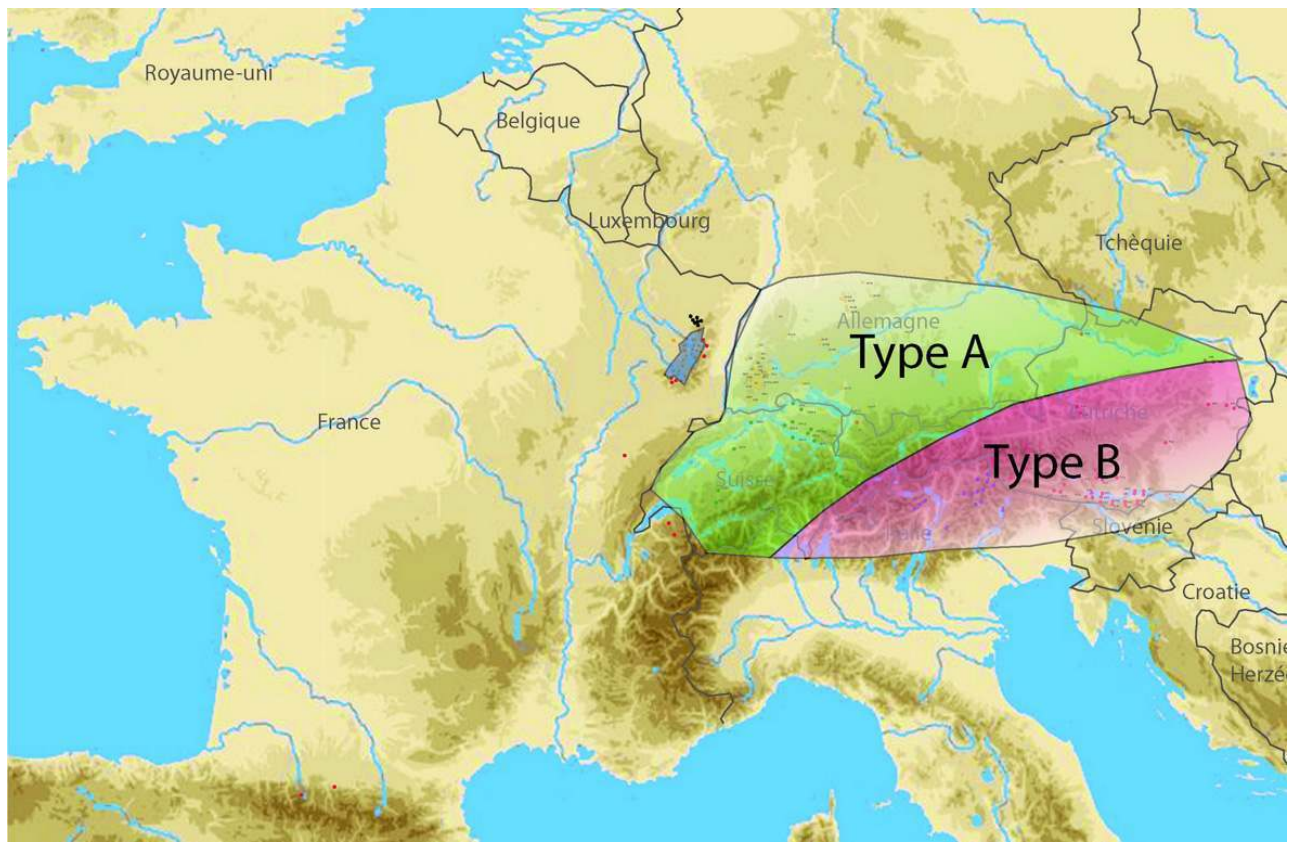

Cartographie de la typologie des scieries d'Europe centrale d'après Herbert Jüttemann. Cartog. Henry, Jean-Yves. (c) Région Lorraine-Inventaire général.

\section{L'évolution des hauts-fers}




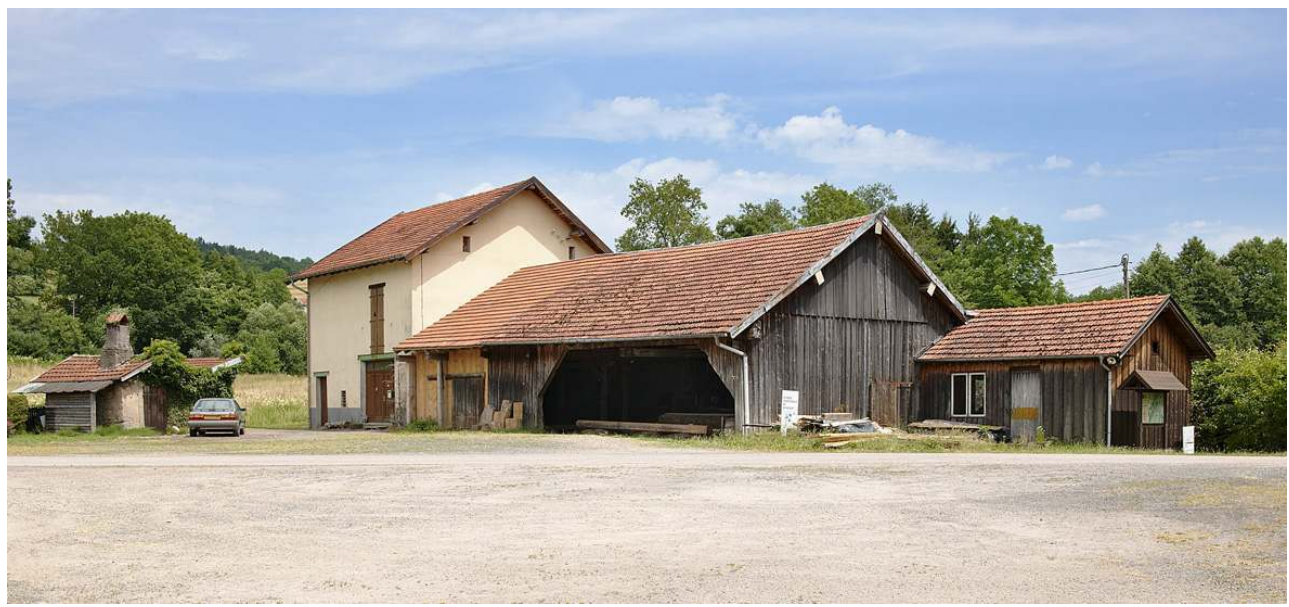

Vue générale de la scierie de Mandray ; de gauche à droite : bâtiment abritant la fontaine et le four à sciure, habitation, scierie, et trieur à grains (Site F).

Phot. Drapier, Bertrand. (Base Mémoire : IVR41_20128810679NUC2A). (C Département des Vosges. C) Région Lorraine-Inventaire général.

13 Les sites de scieries sont généralement aménagés sur un replat de terrain, à la crête duquel la halle de sciage prend place; à l'avant un espace dégagé sert de chargeoir, à l'arrière la dénivellation assure le fonctionnement du système hydraulique (fig. 7). La halle est généralement accompagnée d'une habitation mitoyenne, le logement du sagard ${ }^{6}$, et de petits bâtiments annexes (foyer destiné à brûler la sciure, local d'affûtage des scies, entrepôts...). Les halles, bien que construites sur un sous-sol maçonné, sont essentiellement faites de bois, tant pour la structure du bâtiment, la scie ou la roue hydraulique. Les axes de transmission ou de guidage sont les seules parties qui deviennent métalliques au cours de leur évolution. Le sapin, résineux le plus accessible localement, en constitue la majeure partie ; le chêne y est utilisé parcimonieusement. En fonction de leur usage et de la proximité avec l'eau, certains organes sont remplacés régulièrement. La durée de vie maximale d'une roue hydraulique, l'un des organes les plus fragiles, est estimée à 15 ans. La nécessité de garder un outil performant induit donc une reconstruction régulière (fig. 8, 9, 10). 
Figure 8

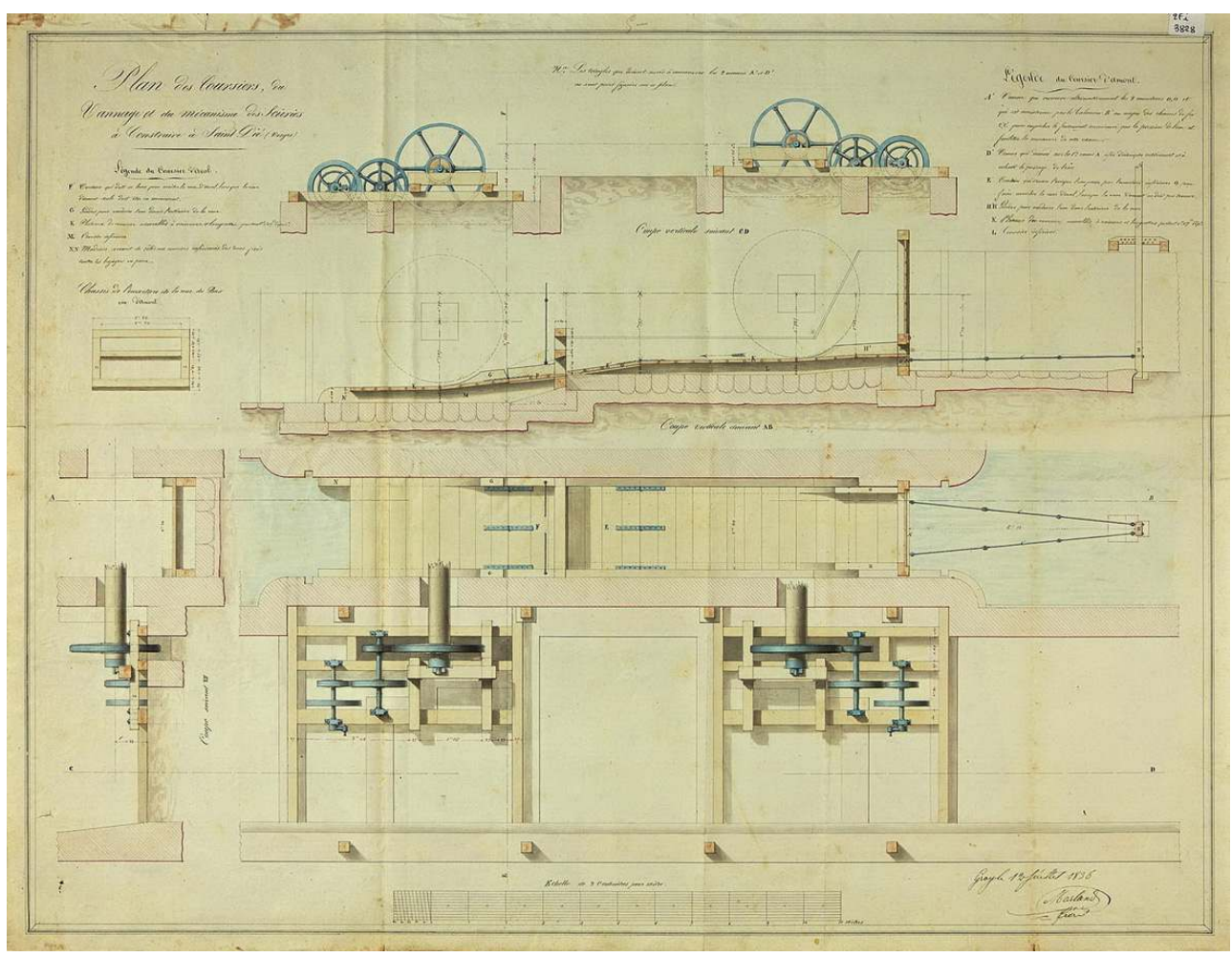

Plans et coupes des coursiers et vannes de la scierie double de Villé à Saint-Dié-des-Vosges. Dessin aquarellé daté de 1836. Sources AD Vosges, 2 Fi 3828. (Base Mémoire : IVR41_20128810186NUC2A). (c) Département des Vosges. (C) Région Lorraine-Inventaire général. 
Figure 9

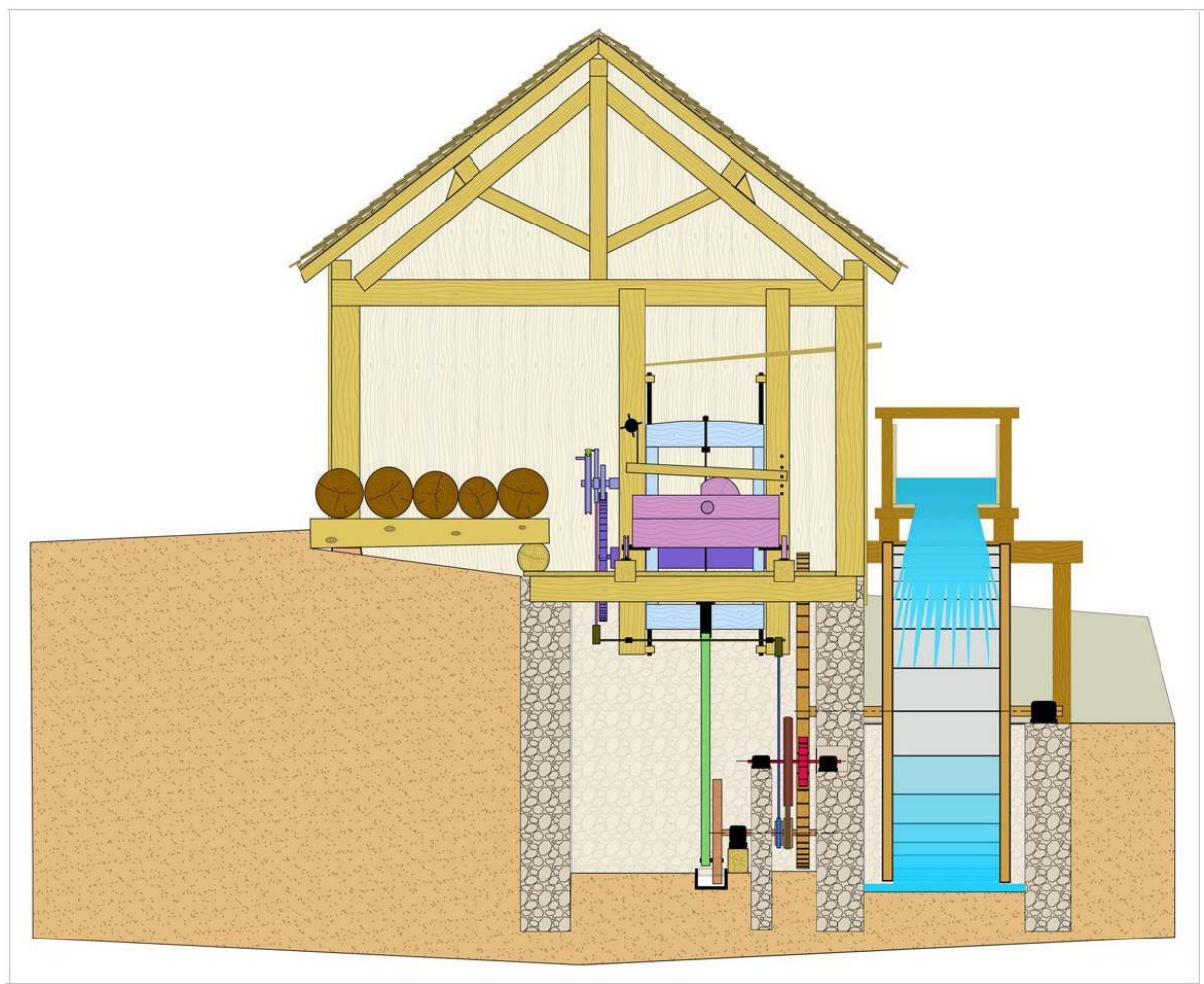

Dessin en transversale d'un haut-fer type avec roue par le dessus.

Dess. Henry, Jean-Yves. (Base Mémoire : IVR41_20118810901NUDA). (c) Région Lorraine-Inventaire général.

Figure 10

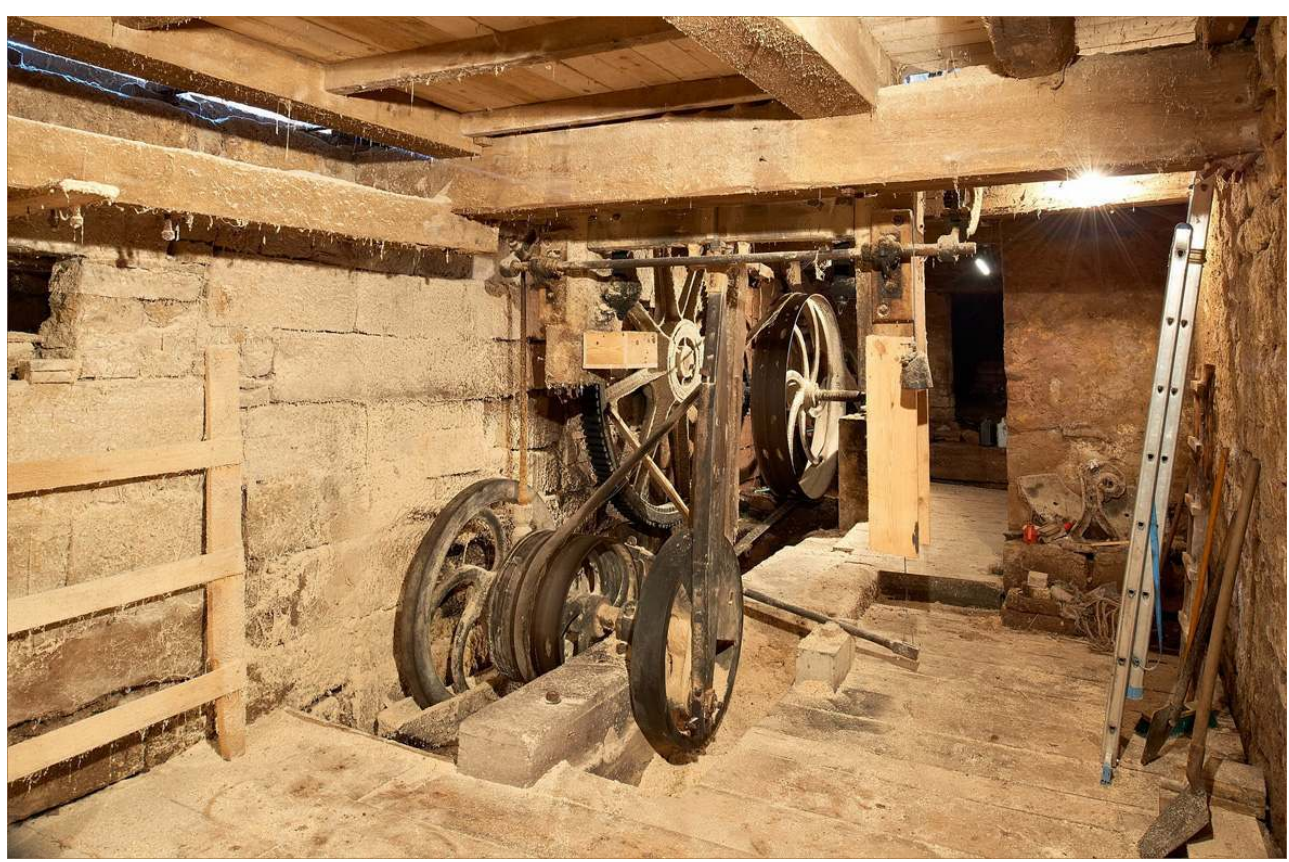

Embiellage et mécanisme multiplicateur de vitesse du haut-fer de Brouaumont à la Houssière (site E). Phot. Drapier, Bertrand. (Base Mémoire : IVR41_20118810306NUC4A). (c) Région Lorraine-Inventaire général. 


\section{La reconstruction de la scierie domaniale de Saint-Marc d'Allarmont ${ }^{7}$}

14 Cette scierie, pour laquelle l'inventaire de 1852 mentionne: «scierie à plomb... avec cheminée dans la cuisine ${ }^{8}$ » fait l'objet d'un rapport du garde des forêts du cantonnement de Raon-l'Étape, en 1868, qui mentionne qu'elle est excessivement endommagée et doit être remplacée immédiatement. Le chariot et la charpente de la halle sont en très mauvais état... Il démontre, chiffres à l'appui, qu'en installant une turbine, la scierie pourrait débiter 4500 planches annuellement au lieu des 2500 actuellement. Elle est entièrement reconstruite en 1869 sur les plans de l'entreprise F. Royer d'Épinal qui y place une turbine de sa fabrication. Incendiée, elle est aujourd'hui en ruine. Seuls la maçonnerie du sous-sol, les éléments métalliques de transmission et la turbine avec sa conduite forcée sont conservés (fig. 11, 12, 13).

\section{Figure 11}

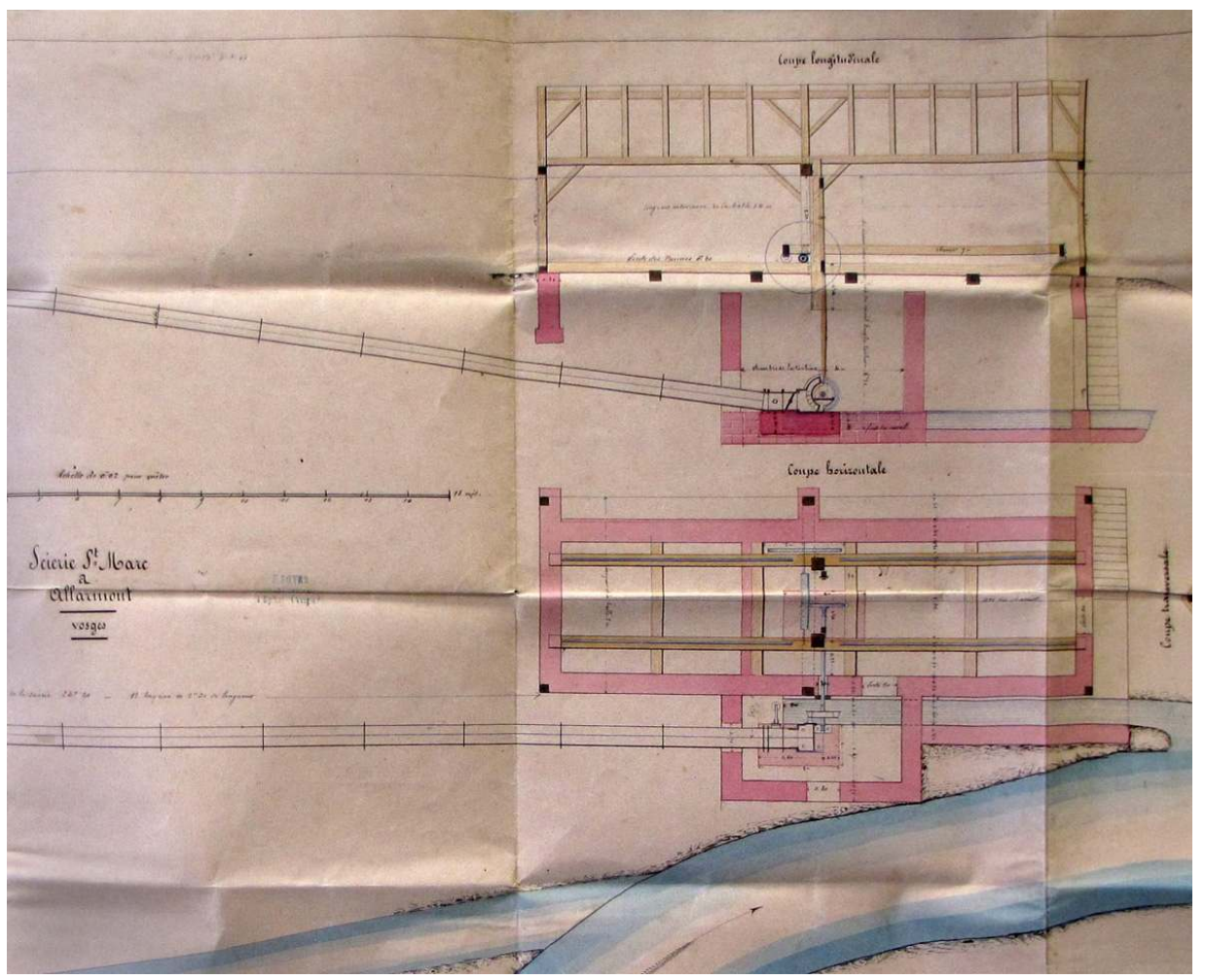

Plans de la scierie domaniale de Saint-Marc d'Allarmont. Extrait de dessin aquarellé daté de 1869. Sources AD Vosges, 2 Fi 5166.

Repro. Henry, Jean-Yves. (c) Département des Vosges. (C) Région Lorraine-Inventaire général. 
Figure 12

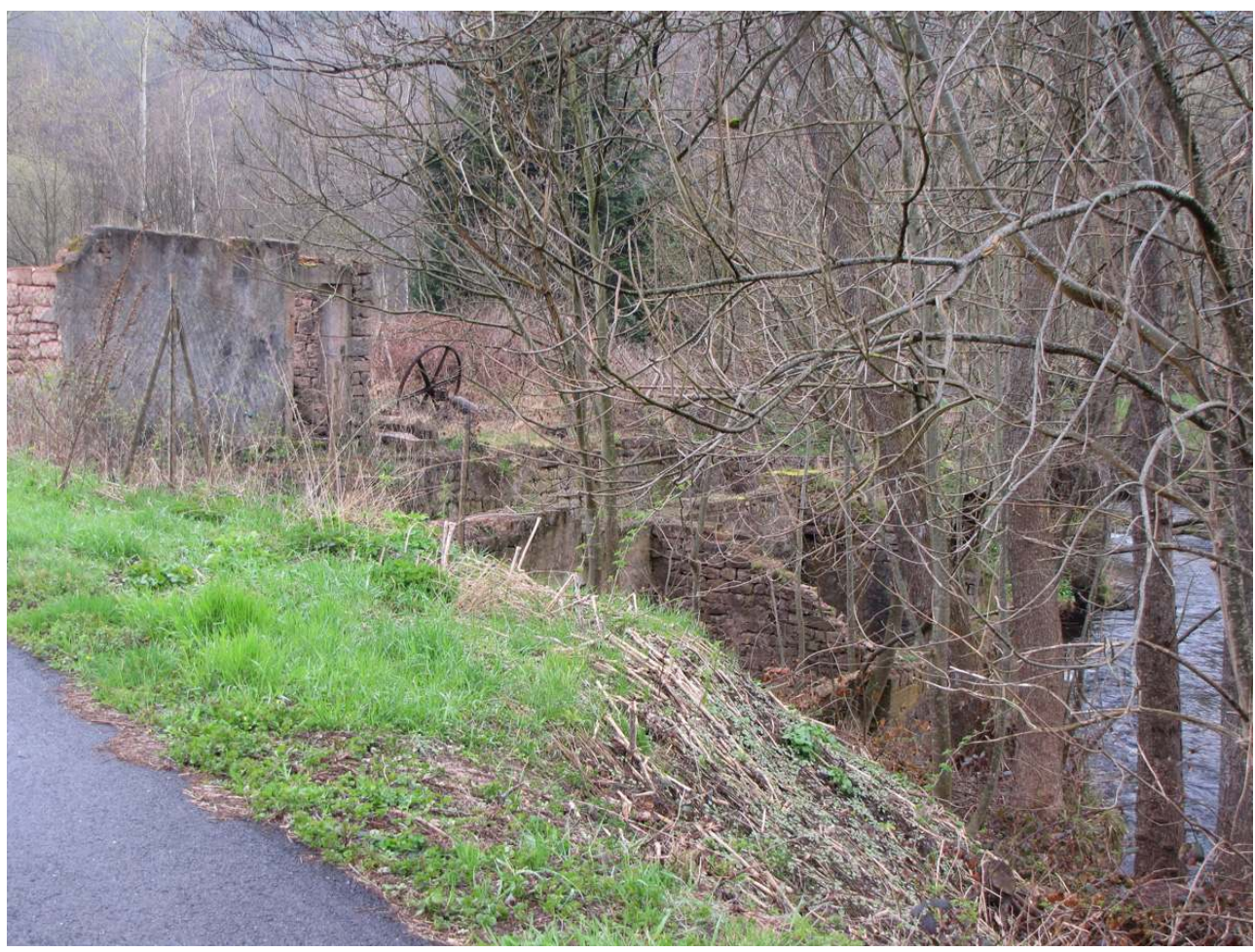

Scierie domaniale de Saint-Marc d'Allarmont, vue générale du site.

Phot. Henry, Jean-Yves. (Base Mémoire: IVR41_20118803141NUC2A). (c) Région Lorraine-Inventaire général. 


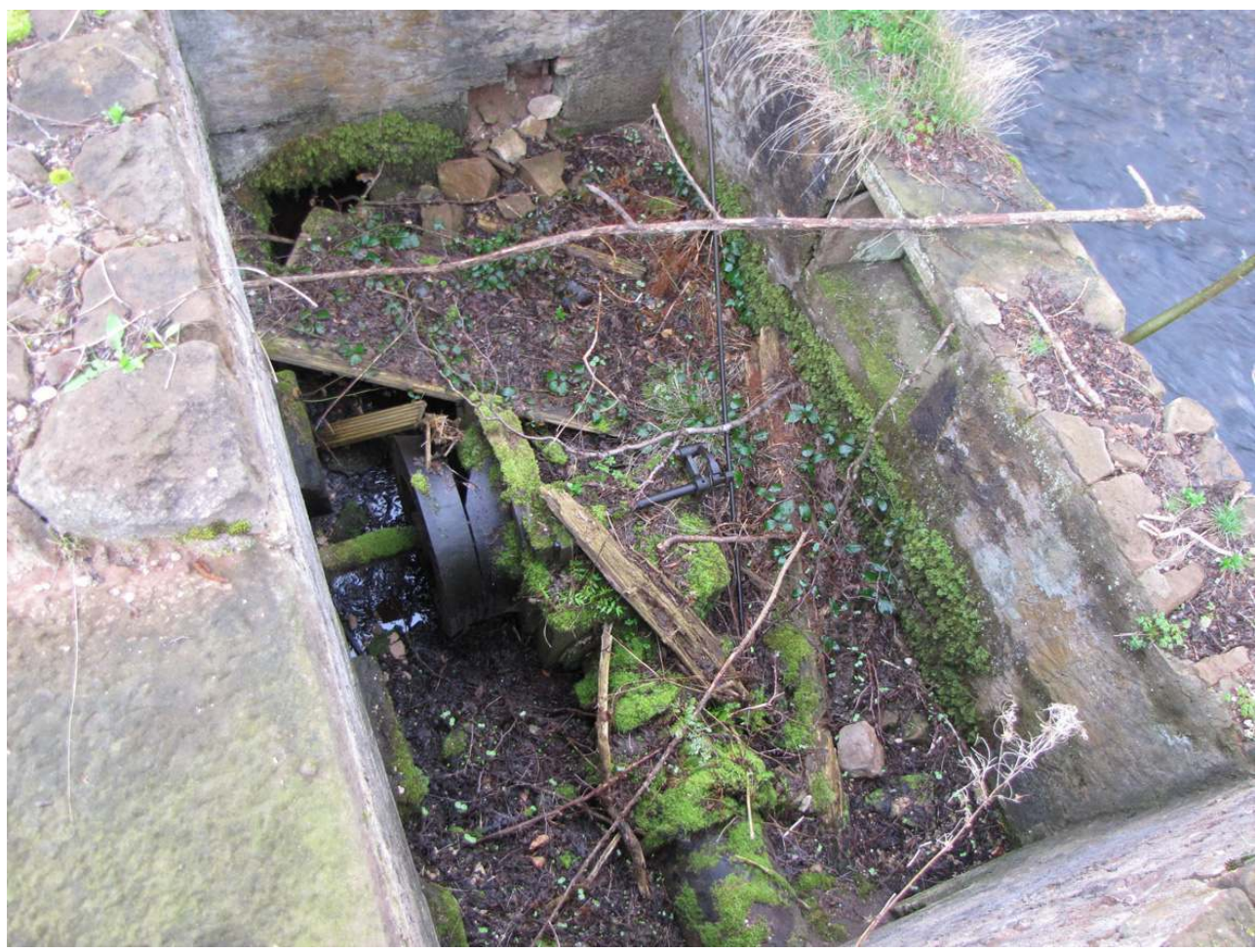

Scierie domaniale de Saint-Marc d'Allarmont, vue de la turbine en situation.

Phot. Henry, Jean-Yves. (Base Mémoire : IVR41_20118803146NUC2A). @ Région Lorraine-Inventaire général.

\section{L'avènement des scieries industrielles}

Dès la fin du $\mathrm{xx}^{\mathrm{e}}$ siècle, la motorisation des scieries évolue; le moteur hydraulique est économique mais trop aléatoire : pris par la glace en hiver et souffrant de manque d'eau en période d'étiage. Les scieries s'équipent souvent d'une seconde source d'énergie, tout d'abord la vapeur puis les moteurs thermiques et enfin les moteurs électriques. Les machines aussi se transforment: des déligneuses circulaires destinées à éliminer les bords bruts des planches, des "rouleaux dédoubleurs" pour refendre les grumes équarries apparaissent à côté du haut-fer. Ils sont encore de fabrication locale et insérés dans un châssis en bois. Au début du $\mathrm{Xx}^{\mathrm{e}}$ siècle arrivent les scies alternatives multiples de construction industrielle mais elles prennent encore place à côté du haut-fer et n'en sont qu'une version industrielle dans un châssis en fonte à plusieurs lames (fig. 14). 


\section{Figure 14}

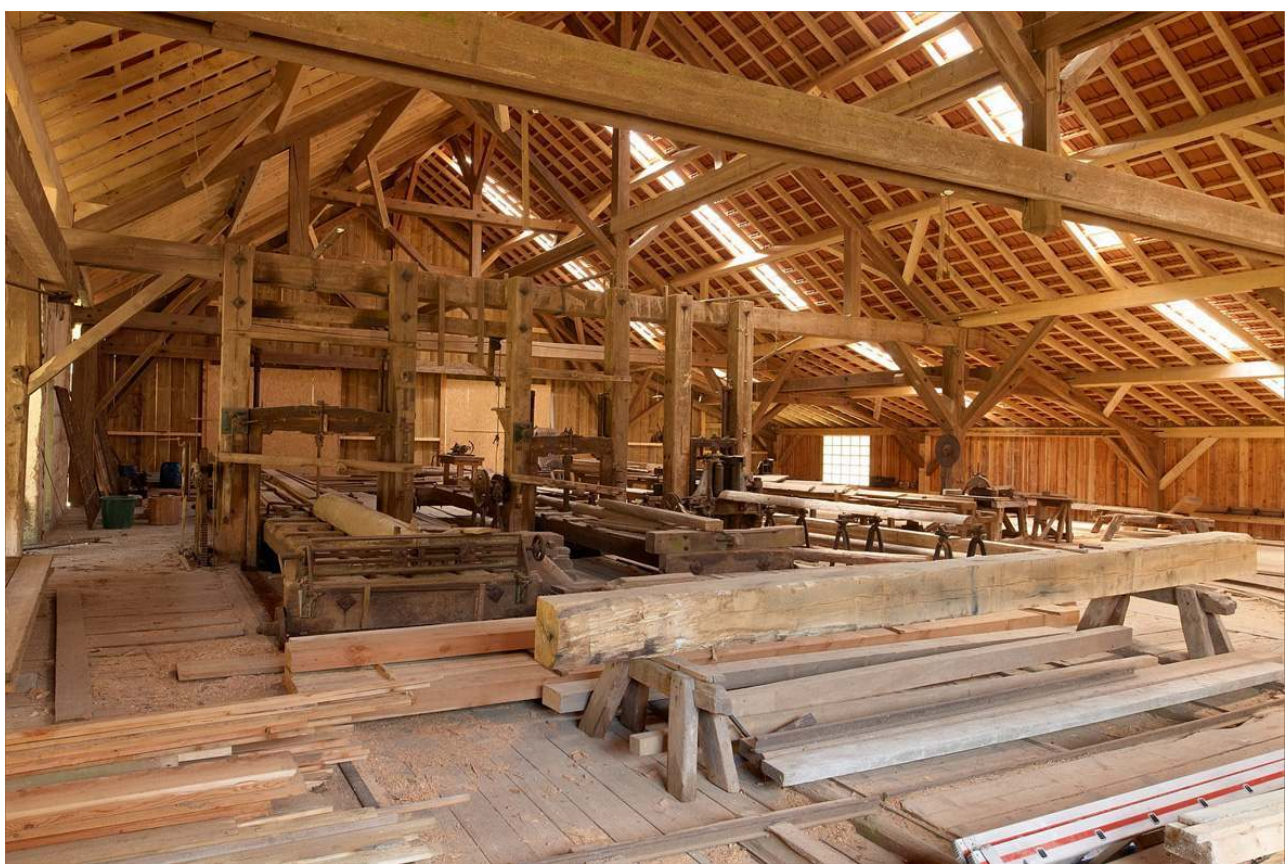

Vue intérieure de la scierie du Fossé à Moussey, équipée d'un haut-fer principal, d'un haut-fer de reprise, d'un rouleau dédoubleur et d'une déligneuse circulaire.

Phot. Drapier, Bertrand. (Base Mémoire : IVR41_20118810431NUC4A). (c) Région Lorraine-Inventaire général.

Finalement, dans les années 1950 commencent à être utilisées les scies à ruban électriques. Les nouvelles scieries sont désormais libérées de la contrainte de l'eau, elles s'installent à proximité des voies de communication, aux abords des villes. Les petites cessent progressivement leurs activités dans les années 1960, entrânant avec elles la disparition du métier de sagard (fig. 15, fig. 16). 


\section{Figure 15}

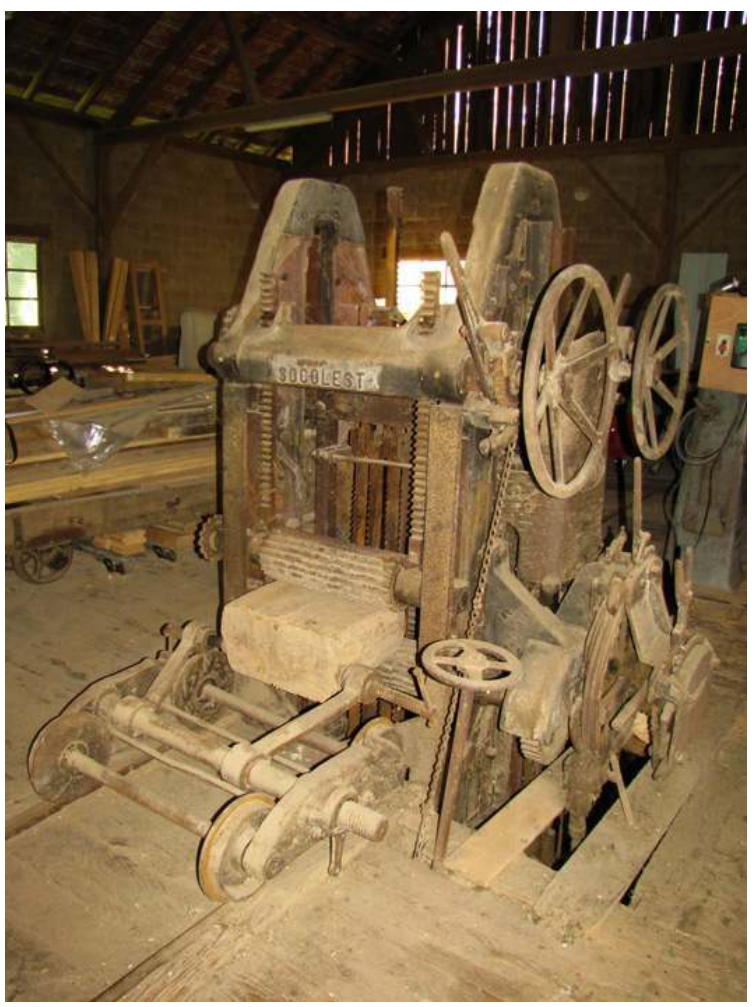

Scie alternative multiple dans la scierie de Vidonchamps aux Rouges-Eaux.

Phot. Henry, Jean-Yves. (Base Mémoire : IVR41_20118803022NUC2A). (C) Région Lorraine-Inventaire général. 


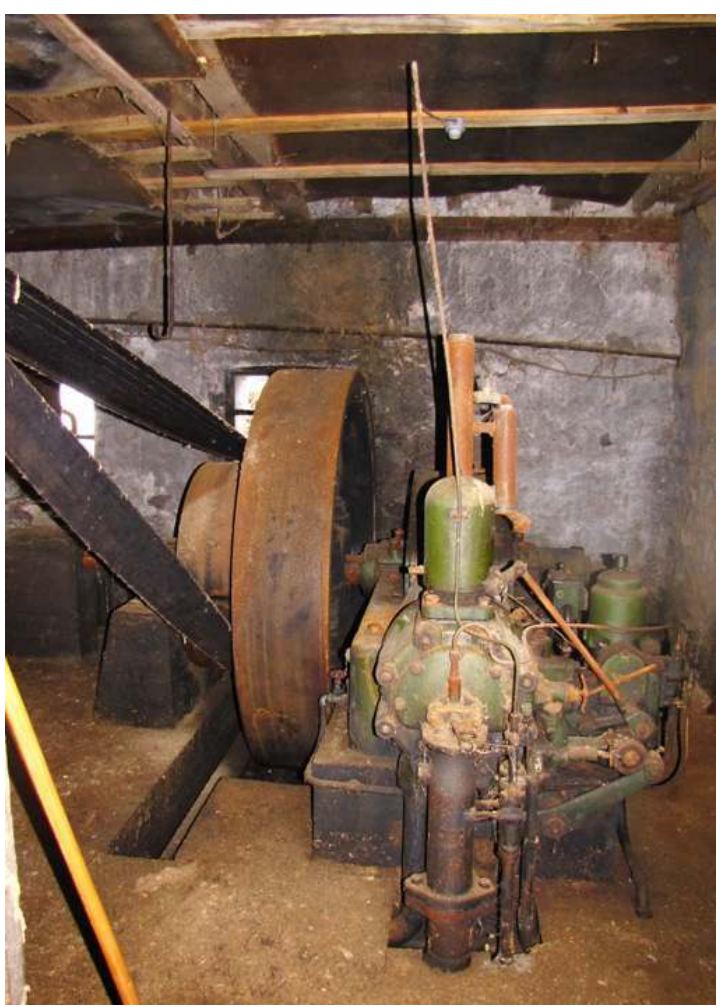

Moteur Ruston \& Hornsby Itd à la scierie de la Forge à Étival-Clairefontaine.

Phot. Henry, Jean-Yves. (Base Mémoire : IVR41_20118803210NUC2A). (c) Région Lorraine-Inventaire général.

\section{Protection, conservation et restauration des sites}

En France, 8 scieries sont protégées en tant que telles au titre des monuments historiques, ( 5 autres le sont en tant que partie constituante du site protégé), 2 sont situées en Franche-Comté, 2 en Alsace et 4 dans les Vosges. La scierie de la Hallière à Celle-surPlaine (site C), seule scierie classée en France, l'a été relativement précocement, en 1978, grâce à la mobilisation d'une association soucieuse de son devenir, désireuse de l'animer et de l'ouvrir au public. En 1982, la scierie Lajus, située sur la même commune (site C) est inscrite car ses installations hydrauliques sont menacées par la création d'un plan d'eau voisin'. La scierie des Fougères (site I) est inscrite en 1986, elle possède une roue hydraulique de 8 mètres de diamètre alimentée par un siphon métallique, hélas détruite par un incendie en 1993, elle est rayée de la liste des monuments historiques en 2001 (fig. 17). La scierie du Lançoir (site B) est inscrite en 1997 (fig. 18). Elle présente l'intérêt d'être l'unique scierie équipée d'une turbine de type Canson encore en état de fonctionnement, d'être régulièrement visitée et de susciter l'intérêt des collectivités locales $^{10}$. Toutes ces scieries sont équipées d'un haut-fer hydraulique. Le dernier site protégé, le moulin-scierie-saboterie Thiriet à Xamontarupt (site H), l'est en 2013 (fig. 19). Il a été sélectionné à l'issue de l'étude d'inventaire de la petite industrie hydraulique, sur des critères qui le démarquent des autres sites : sa polyactivité. Il s'organise autour d'une ferme et conserve dans différents bâtiments une minoterie encore équipée d'une paire de 
meules, une saboterie et une scierie à scie alternative de côté de construction artisanale. Il est animé par une association.

Figure 17

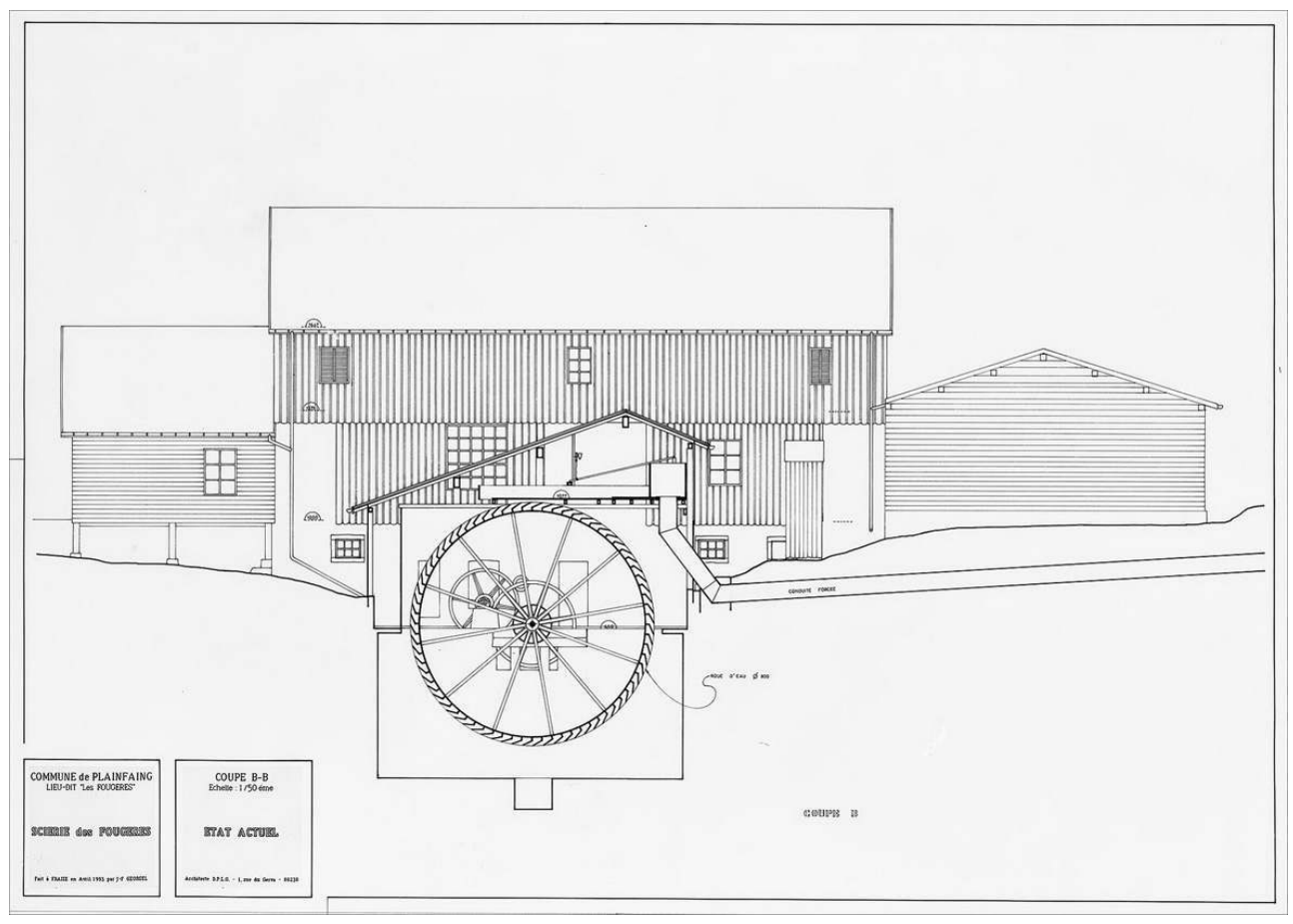

Plan de la scierie des Fougères à Plainfaing (site I).

Dess. Georgel, Jean-François. (Base Mémoire : IVR41_948800129P). @ Région Lorraine-Inventaire général. 
Figure 18

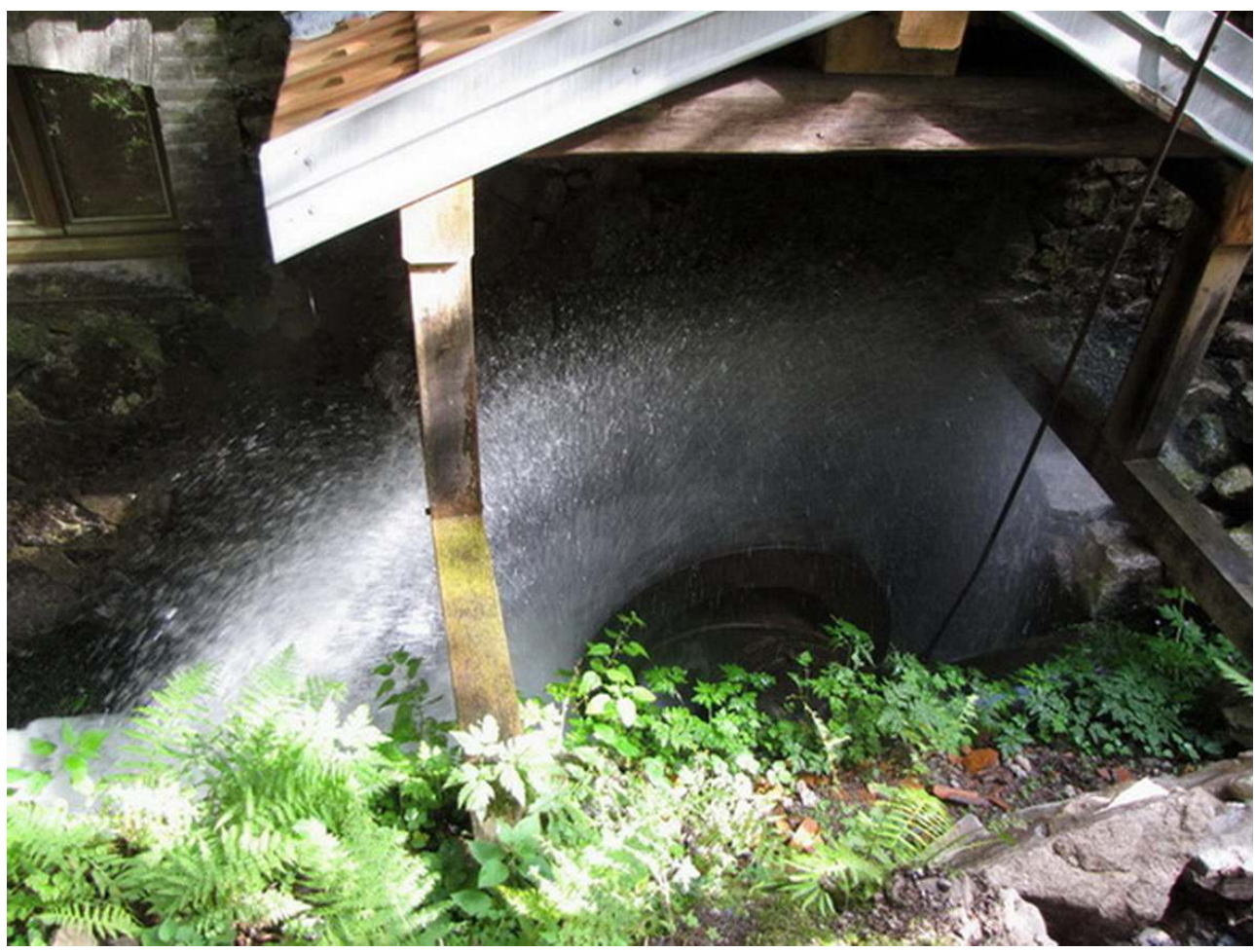

Turbine hydraulique de la scie du Lançoir à Ban-sur-Meurthe-Clefcy (site B).

Phot. Henry, Jean-Yves. (c) Région Lorraine-Inventaire général.

Figure 19

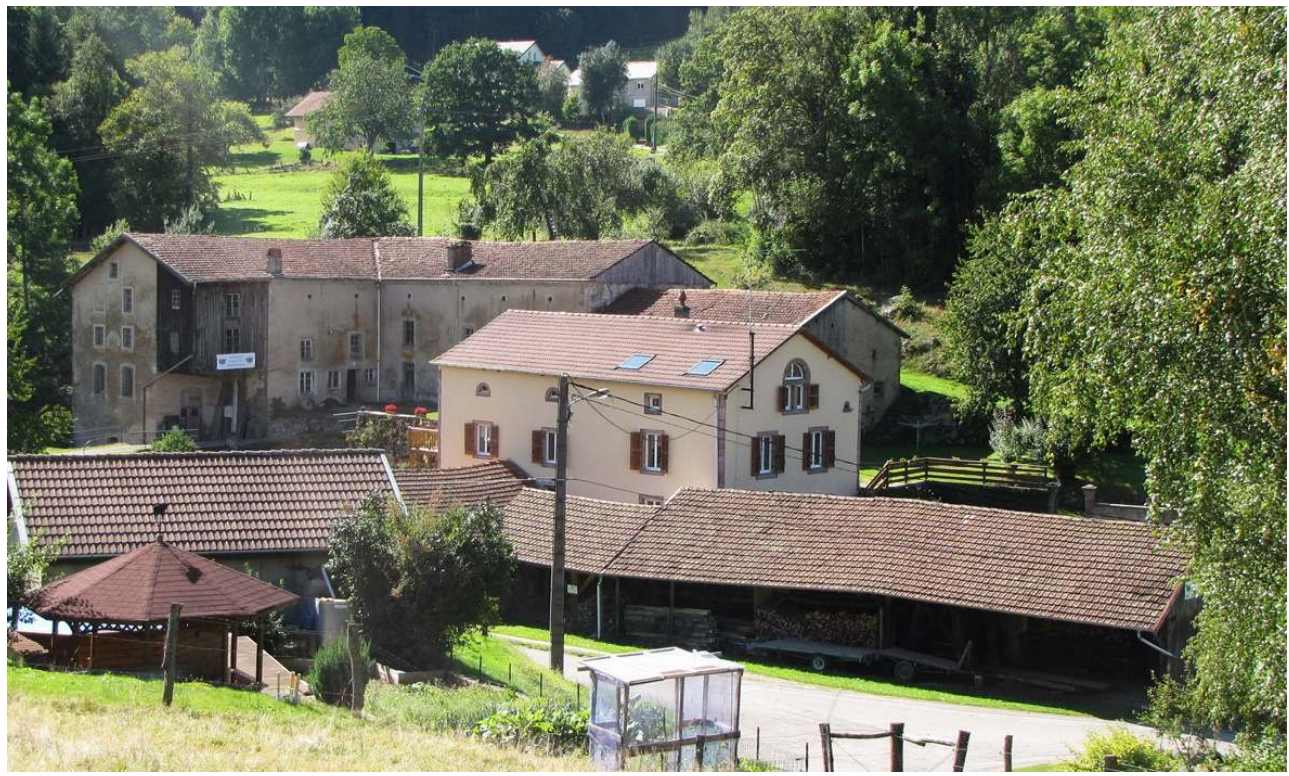

Vue générale du moulin Thiriet à Xamontarupt (site H).

Phot. Henry, Jean-Yves. (Base Mémoire : IVR41_20118803295NUC2A). @ Région Lorraine-Inventaire général.

18 Plusieurs autres sites présentent un intérêt majeur dans l'histoire de l'exploitation forestière vosgienne. Ils pourraient être proposés à une protection au titre des 
monuments historiques, mais comment l'envisager aujourd'hui sans une volonté manifeste de leur propriétaire, un état correct des bâtiments et des machines et un projet de valorisation? L'histoire des sites le prouve, la protection d'une scierie ne peut se concevoir sans prévoir un entretien régulier des bâtiments. Comment sans cela lutter contre les dégradations d'un bâtiment à structure en bois qui a les pieds dans l'eau, ou plus simplement éviter l'incendie d'un bâtiment en bois rempli de sciure, deux bâtiments protégés ont été ainsi détruits! (la Hallière, site $C$, et les Fougères, site I). De plus, l'absence d'entretien des biefs et des vannes peut provoquer soit une inondation, soit un manque d'eau, deux incidents préjudiciables à la bonne conservation du site.

L'autre question concerne les restaurations, qu'elles soient partielles ou totales. Comment trouver aujourd'hui des artisans capables de reconstruire une roue hydraulique ou une partie du mécanisme et ayant conservé un savoir-faire local ? Tous les ateliers spécialisés dans ce domaine ont disparu. Les propriétaires sont donc contraints de recourir à une entreprise utilisant des techniques de fabrication de haut niveau qui, faute d'expérience dans ce domaine précis, se limite à reproduire fidèlement les éléments détruits, sans forcément maîtriser les tenants et aboutissants de cette restauration.

\section{La reconstruction de la scierie de la Hallière}

Cette scierie classée a été détruite par un incendie en 2001, sa reconstruction est exemplaire dans ce domaine. Seules les maçonneries du bâtiment ont pu être conservées. Deux scies similaires à celles qui l'équipaient (haut-fer et rouleau dédoubleur) ont été récupérées dans une autre scierie à l'abandon et adaptées au bâtiment reconstruit. Le chantier a été conduit par l'architecte en chef des Monuments historiques Pierre Bortolussi, le coût de la reconstruction s'élève à $620000 €$, financé à $40 \%$ par la communauté de communes, à $25 \%$ par l'État, à $23 \%$ par le département et à $12 \%$ par la région. Elle est ré-ouverte au public depuis 2013 (fig. 20, fig. 21). 
Figure 20

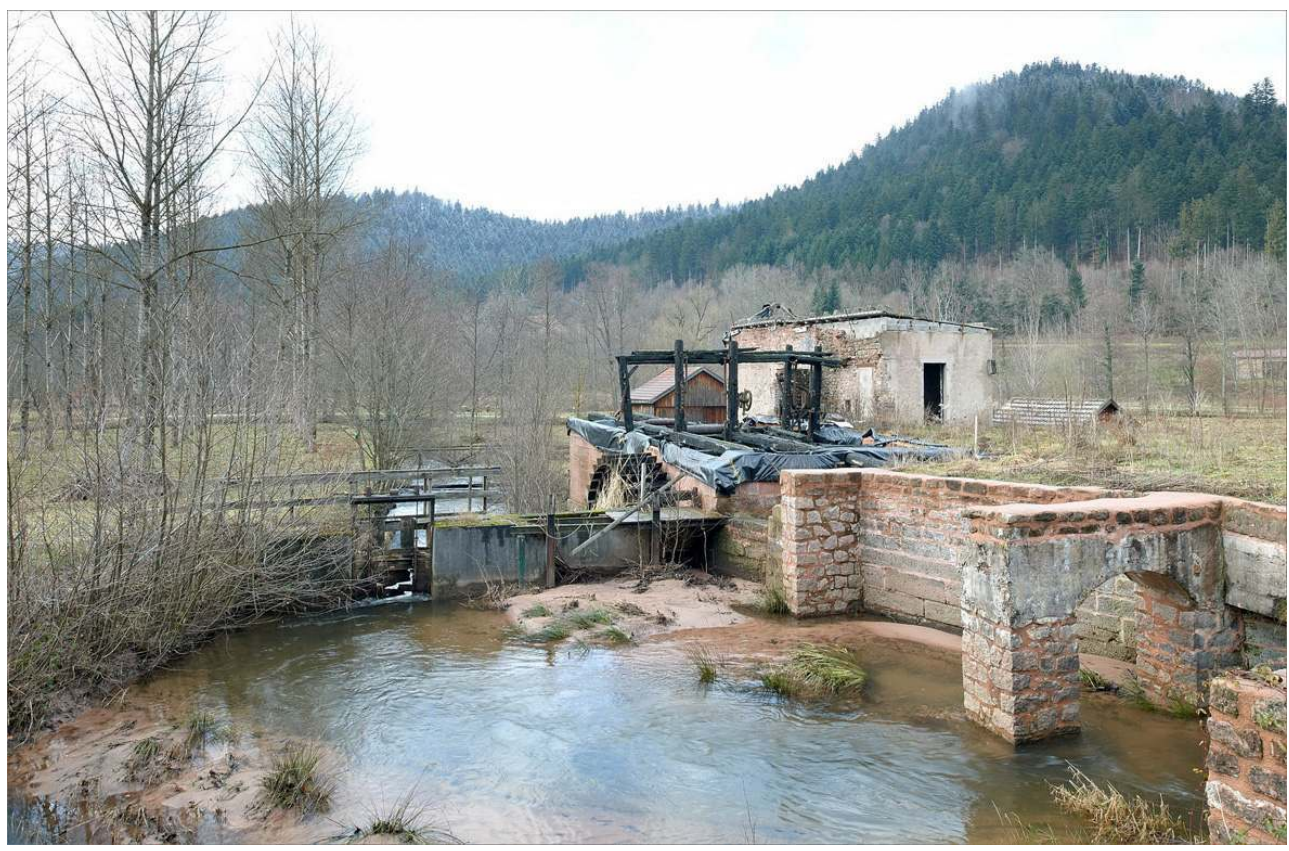

Vue d'ensemble de la scierie détruite de la Hallière à Celles-sur-Plaine (site C).

Phot. Drapier, Bertrand. (Base Mémoire : IVR41_20118810221NUC4A). @ Région Lorraine-Inventaire général.

Figure 21

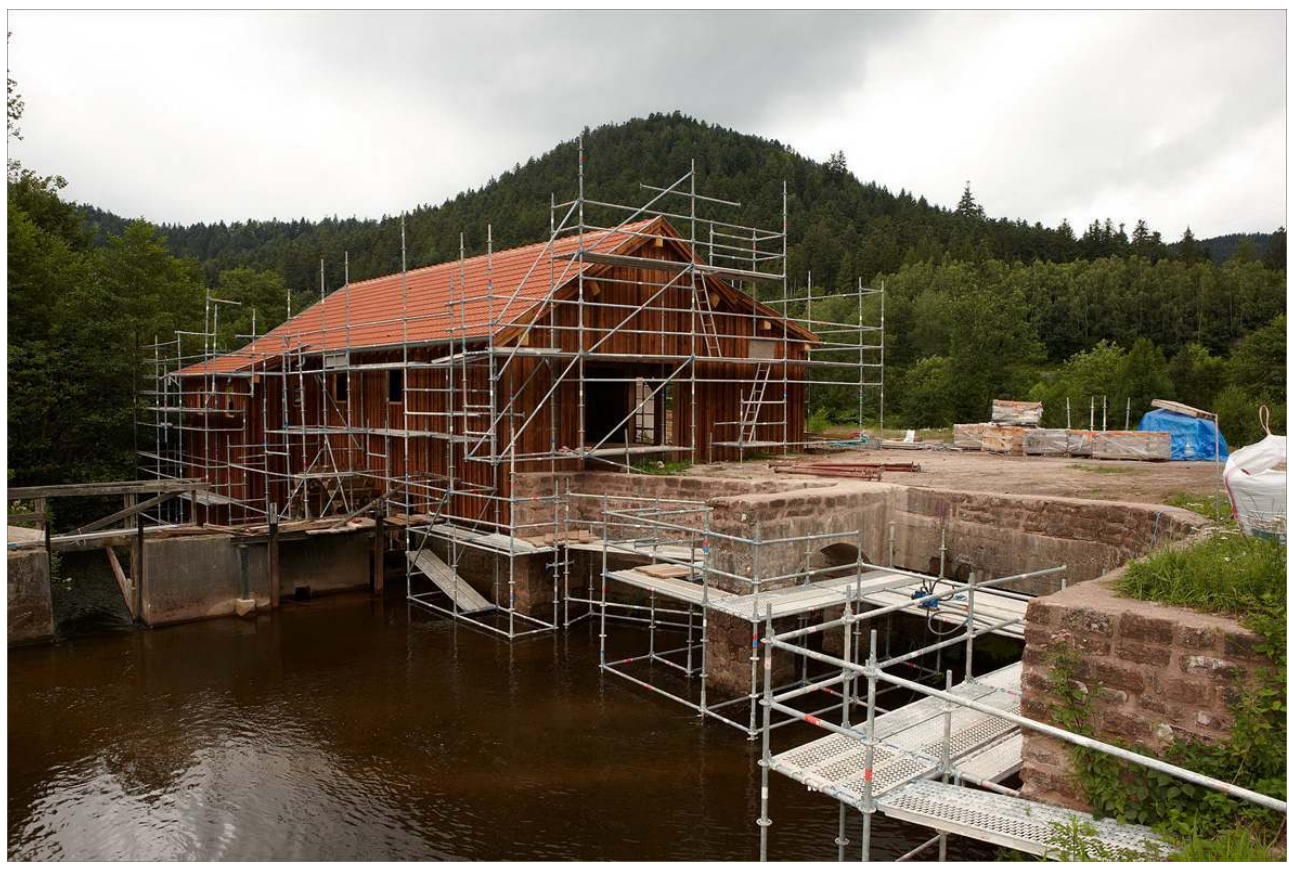

Vue d'ensemble de la scierie de la Hallière à Celles-sur-Plaine (site C) en cours de reconstruction. Phot. Gury, Ludovic, Henry, Jean-Yves. (Base Mémoire : IVR41_20128812060NUC2A). @ Région Lorraine-Inventaire général.

21 Les mesures de protection concernent systématiquement le bâtiment, les aménagements hydrauliques et les machines immeubles par destination. Ces mesures conservatoires 
visent à garder un site en état potentiel de fonctionnement, mais elles seront, rappelonsle, sans effet sans la volonté et la capacité du propriétaire de les maintenir en état. On comprend aisément que cet effort perpétuel de conservation ne peut se concevoir sans un projet de valorisation qui permette au propriétaire de trouver les ressources nécessaires à l'autofinancement des travaux réguliers.

\section{La protection des machines et du matériel} site, qui ne lui est généralement pas lié historiquement, même si sans appareillage ni outillage, la compréhension du fonctionnement d'une scierie est pour le moins problématique.

sagard, locataire de la scierie, à l'image du meunier, était tenu «de pourvoir les scieries de tous les outils ou accessoires nécessaires au roulement de ces usines, tels que hauts fers, paufers, masses de fer, limes, lames, meules à émeri, lanternes, cordes, cordeaux, huile, graisse, etc.", comme le précisent les différents «règlement des scieries » établis par l'administration des Eaux et Forêts entre 1896 et $1936^{11}$.

Cet ensemble se compose d'une part du matériel spécifique au fonctionnement du hautfer : lames de scie, cales de réglages, griffes de fixation, cordeaux et de produits courants nécessaires à son entretien : graisse, huile, éclairage et limes d'affûtage et d'autre part d'outils destinés à la manipulation des grumes ou du bois scié : crochet à anneau, cric de forestier, pic à grume, scie passe-partout, hache d'abattage, merlin..., outils standards, répandus dans toute l'Europe. Le sagard est donc propriétaire de la totalité des outils et lorsque son contrat se termine, il quitte la scierie avec eux.

orisation d'un site, souvent après des dizaines d'années d'abandon, commence par la reconstitution d'une collection d'outils, parfois rachetés à un ancien sagard, ou le plus fréquemment, patiemment acquis au fil des ans sur les marchés aux puces...

Cette collection est couramment employée lors des démonstrations de sciage afin de faire comprendre au public comment un seul homme peut faire fonctionner la scierie, charger les grumes sur le chariot de sciage et évacuer les planches sciées... Le plus souvent, les sites optent pour la constitution d'une seconde collection d'outils destinés uniquement à être exposés.

On peut difficilement envisager une protection au titre des objets mobiliers de l'ensemble de l'outillage d'une scierie, car pris individuellement, chacun a peu de valeur intrinsèque ou historique. Seule la collection présente un intérêt ${ }^{12}$ et celle-ci ne prend de sens que parce qu'elle est conservée in situ et en état d'être utilisée.

Autre exemple témoin de la complexité d'une politique de protection et de sauvegarde d'objet mobilier : la turbine de la scierie Saint-Marc d'Allarmont (fig. 22). Elle est le seul élément intéressant qui subsiste de cette scierie incendiée, mais elle gît au fond de la ruine et ne va tarder à disparaître définitivement sous les alluvions et la végétation. Cette turbine $^{13}$ en fonte de fer moulé à axe horizontal, centrifuge, à admission axiale, à injection partielle et échappement libre est, à ce jour, un des derniers exemplaires conservés de ce type de turbine. De plus, son principe reste parfaitement inconnu au niveau national, n'ayant fait l'objet d'aucun brevet ni publication ${ }^{14}$. Sa conservation in situ serait une aberration à long terme. Sa seule chance de sauvegarde serait de trouver une structure muséale qui accepte de la prendre en charge, condition préalable à sa 
protection. Elle ne pourrait alors qu'être présentée dénuée de tout contexte naturel et technique.

Figure 22

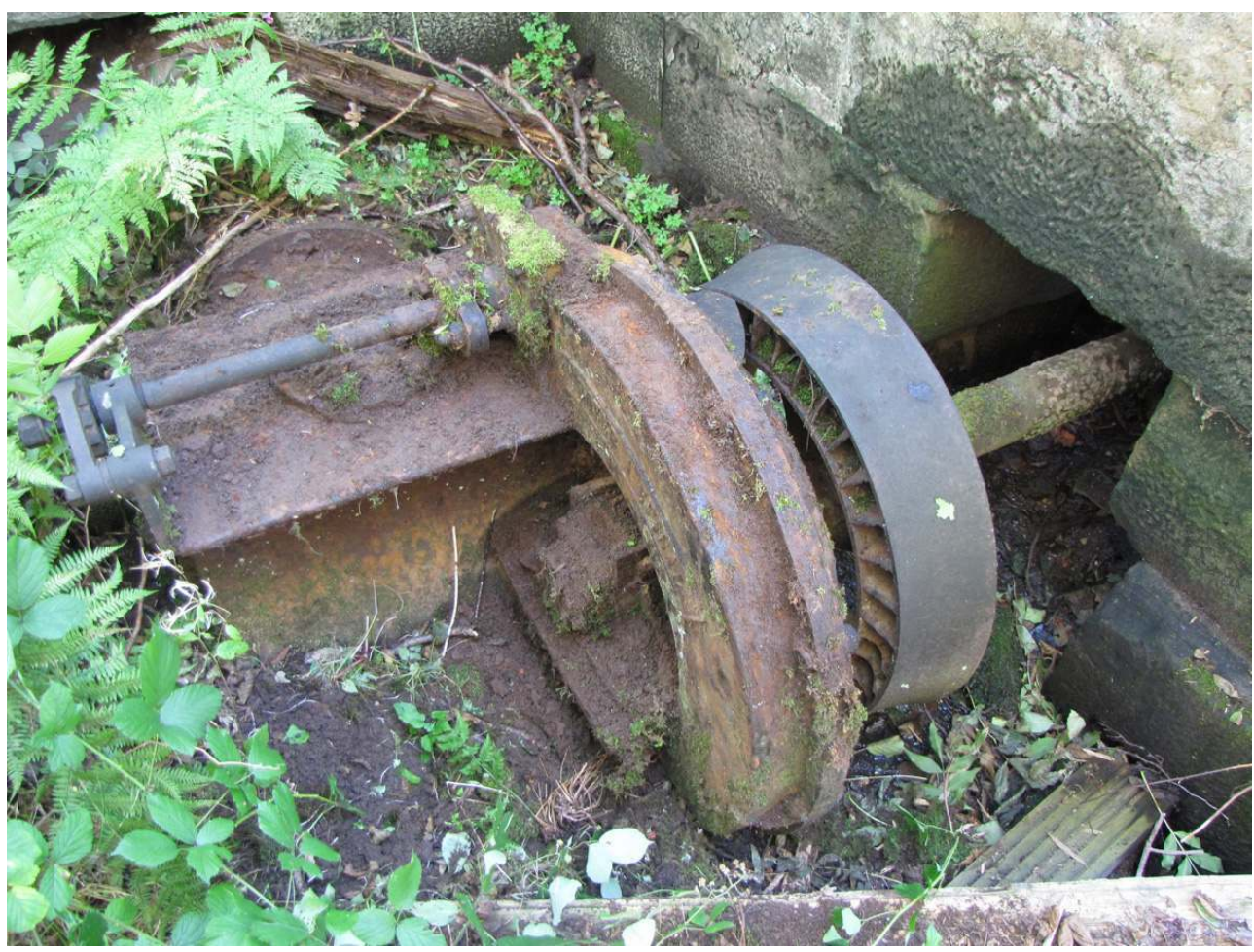

Vue de la turbine de la scierie Saint-Marc d'Allarmont en situation.

Phot. Henry, Jean-Yves. (Base Mémoire : IVR41_20118803136NUC2A). @ Région Lorraine-Inventaire général.

Plusieurs autres types de turbine à axe horizontal ont été développés spécifiquement pour les scieries et autres petits ateliers par des fonderies locales qui ont aujourd'hui totalement disparu sans même laisser d'archives ${ }^{15}$.

\section{Valorisation}

La majorité des scieries ouvertes au public sont aujourd'hui des propriétés de collectivités locales (communes ou communauté de commune) gérées par des associations loi de 1901. Initialement propriétés privées animées par des bénévoles, les collectivités locales désireuses de pérenniser la valorisation de leur territoire s'en sont portées acquéreuses ${ }^{16}$. Pour les associations gestionnaires, c'est l'occasion de trouver un souffle nouveau auprès d'un partenaire prêt à investir dans l'attractivité du site, mais avec pour corollaire la nécessité, rentabilité oblige, d'accueillir un public plus nombreux. Les situations sont diverses, et les conditions de visite fort variables, en quantité comme en qualité.

Dans la configuration minimale, le propriétaire fait lui-même des visites commentées avec démonstration de sciage sur demande, quelques fois par an (site A). À l'autre extrémité, une scierie salarie un emploi et demi pour accueillir plus de dix mille visiteurs par an avec une ouverture de plus de 11 mois par an et de 5 à 7 jours par semaine (site B). Entre ces deux extrêmes, toutes les variations sont possibles. 

sécurisé de visite, d'une salle d'accueil, d'une billetterie, d'une exposition. Dans le cas d'un édifice protégé, le service territorial de l'Architecture et du Patrimoine veille à éviter toute dénaturation des bâtiments comme du site (fig. 23).

Figure 23

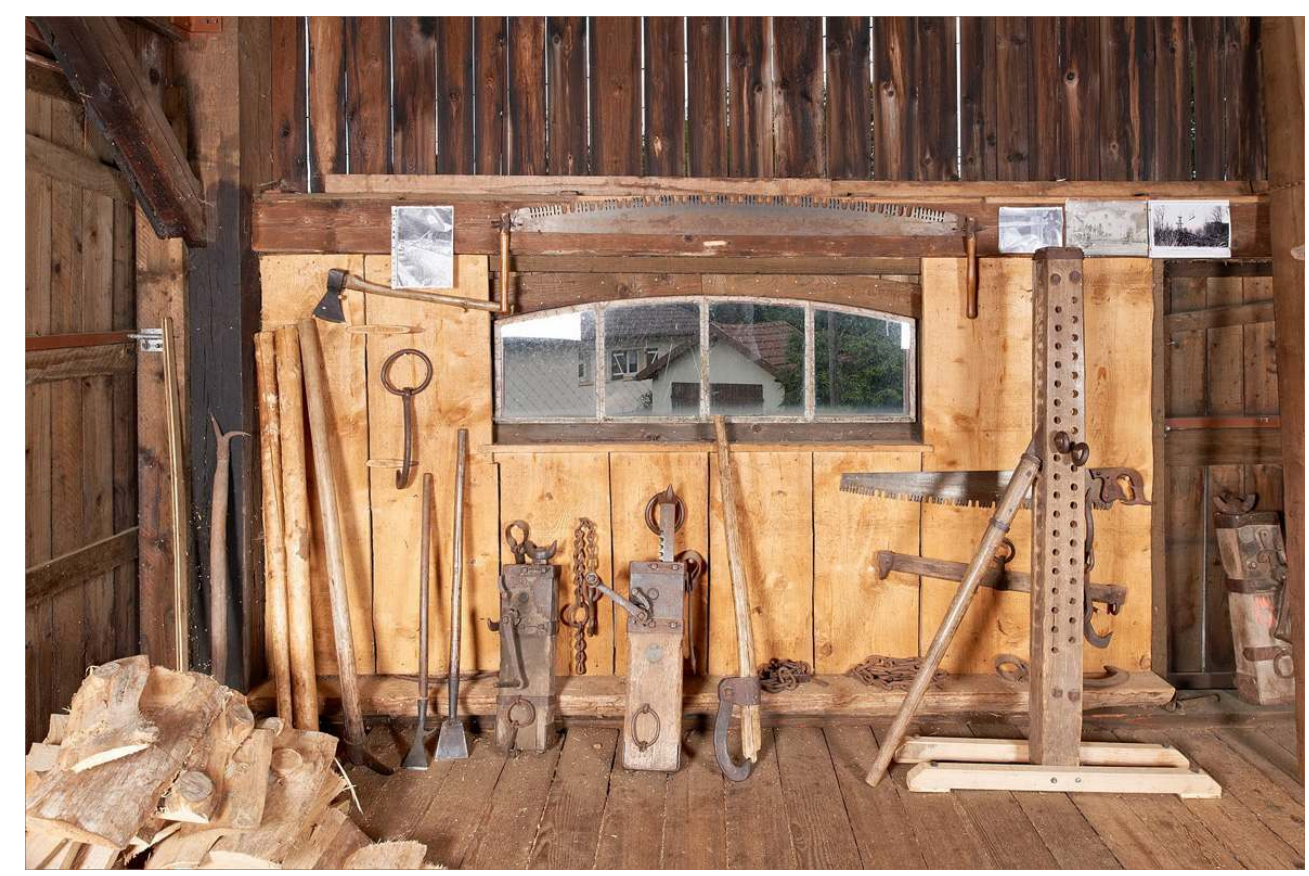

Exposition d'outils au musée du bois à Saulxures-sur-Moselotte (site G).

Phot. Drapier, Bertrand. (Base Mémoire : IVR41_20118810525NUC4A). C Région Lorraine-Inventaire général.

Le nombre de visiteurs de tels sites n'étant pas extensible, certaines scieries recherchent une clientèle locale ou plus ciblée. La scierie de Mandray (site F) a fait le choix de transformer un garage automobile adjacent en salle d'accueil destinée principalement à un public scolaire (fig. 24). Les expositions ont été élaborées en partenariat avec le service régional de l'Inventaire général et l'une d'elles rend compte des résultats de l'enquête sur les scieries hydrauliques. 


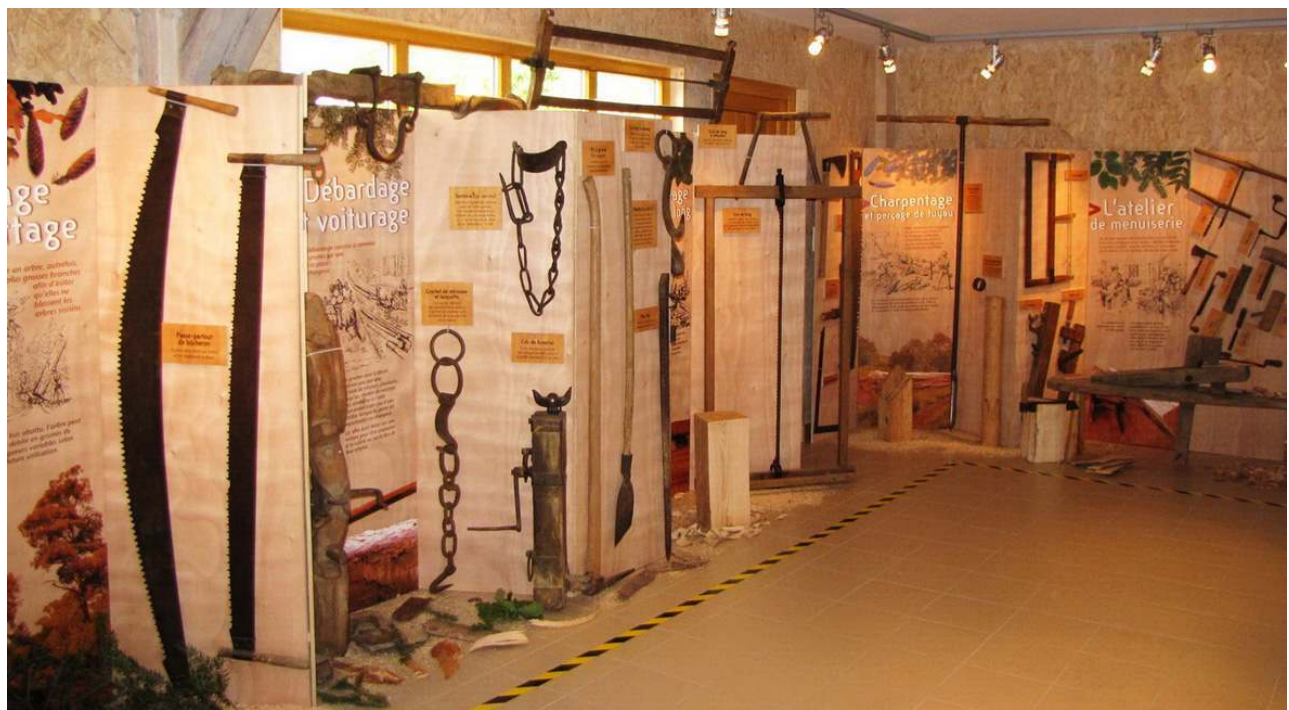

Expositions présentées dans la salle d'accueil de la scierie communale de Mandray (site F).

Phot. Henry, Jean-Yves. (c) Région Lorraine-Inventaire général.

\section{Liste des scieries protégées ou ouvertes au public et en état de fonctionnement}

Voir le tableau en annexe

\section{BIBLIOGRAPHIE}

ARMENGAUD aîné [Jacques-Eugène]. Traité théorique et pratique des moteurs hydrauliques. Paris : chez l'auteur, 1858.

BELIDOR, Bernard FOREST de. Architecture hydraulique, ou L'art de conduire, d'élever et de ménager les eaux pour les différens besoins de la vie [1737-1739]. Paris : Jombert, 1782-1789, Première partie, tome premier.

BELHOSTE, Bruno, LEMAÎTRE, Louis. «J.V. Poncelet, les ingénieurs militaires et les roues et turbines hydrauliques ». Cahier d'histoire des sciences et des techniques, ${ }^{\circ} 29,1990$, p. 33-89. BOILEAU, Pierre-Prosper. Instruction pratique sur les scieries [1855]. Paris : E. Lacroix, 1861. BOITHIAS, Jean-Louis, BRIGNON, Marc. Les Scieries et anciens sagards des Vosges. Bûcherons, schlitteurs, voituriers et voileurs. Nonette : Créer, 1985.

JÜTTEMANN, Herbert. Alte Bauernsägen im Schwarzwald und in den Alpenländern. Karlsruhe : Braun, 1984. 
LAURENT, Paul. Précis du cours de constructions forestières à l'École royale forestière de Nancy

[1844-1846]. Nancy : chez l'auteur, 1848.

REGNEAULT, Étienne-Emmanuel. Traité de mécanique comprenant les premiers éléments de la science des machines et leur application aux scieries forestières. Nancy : Grimblot, Vve Raybois et Cie, 1857.

VIOLLET, Pierre-Louis. Histoire de l'énergie hydraulique. Moulins, pompes, roues et turbines de l'Antiquité au XX siècle. Paris : Presses de l'École nationale des ponts et chaussées, 2005.

\section{NOTES}

1. - Activité de taille des roches dures, telles le granite.

2. - Le principe communément adopté en Europe consiste à effectuer le sciage lorsque le chariot est à l'arrêt. La lame dont les dents sont dirigées vers le bas et dont le haut est légèrement incliné vers l'avant, scie en descendant et le chariot avance lorsque la lame remonte.

3. - Scierie double du moulin de Villé à Saint-Dié-des-Vosges (IA88001782).

4. - Archives départementales des Vosges (AD), ONF cantonnement de Raon-l'Étape, archives en cours de classement.

5. - Herbert Jüttemann, dans Alte Bauernsägen im Schwarzwald und in den Alpenländern (Karlsruhe : Braun, 1984), étudie et répertorie une centaine de scieries dont il détaille les principes et techniques.

6. - Sagard: de l'allemand sägen = scier, le scieur responsable d'une scierie à haut-fer. Il est généralement locataire de la scierie et se rémunère par une commission sur le bois scié. Il loge sur place et a souvent une activité secondaire d'éleveur de bétail.

7. - Voir dans la base Mérimée : notice IA88001784.

8. - AD Vosges, ONF cantonnement de Raon-l'Étape, archives en cours de classement.

9. - Avis sur la protection, Conservation régionale des monuments historiques (CRMH) de Lorraine.

10. - Avis du recenseur, CRMH de Lorraine.

11. - Extrait des différents « règlement des scieries » de 1891, 1920, 1936 (AD Vosges, 47 M 159).

12. - La réflexion en cours dans le cadre de la loi patrimoine pourrait permettre une avancée en proposant une protection comme collection.

13. - Voir dans la base Palissy : notice IM88004724.

14. - Information communiquée par Serge Benoit, spécialiste des turbines au Conservatoire national des Arts et Métiers à Paris.

15. - Beyer frères à Saint-Dié, Wiedemann à Rothau, Duchêne à Fresse-sur-Moselle, plus connues : Altoffer à Remiremont, Royer et Joly à Épinal, Négri à Saint-Dié et Singrün à Golbey.

16. - Le moulin-scierie-saboterie de Xamontarupt a fait l'objet d'une étude de faisabilité pour sa valorisation touristique (cofinancée par la région Lorraine) dans l'éventualité de son rachat par la communauté de communes de la vallée de la Vologne. 


\section{RÉSUMÉS}

Dans le cadre de l'étude thématique de la petite industrie hydraulique de la montagne vosgienne, le service de l'Inventaire général de la région Lorraine a entrepris l'étude des scieries hydrauliques. Plus de 250 sites sont signalés dans les archives, 115 sites allant du vestige à la scierie complète ont été repérés sur le terrain. 25 ont fait l'objet d'une monographie publiée dans la base Mérimée et un a été inscrit au titre des monuments historiques pour compléter le corpus déjà protégé. Certains sites sont connus de longue date, classés ou inscrits, exploités touristiquement. D'autres, bien qu'ayant conservé leur machinerie d'origine, peuvent être à l'état d'abandon complet, ou chose plus rare, restaurés par leur propriétaire privé et conservés à l'abri des regards indiscrets. D'autres encore ont subi les multiples vicissitudes d'un bâtiment technique, sans cesse transformé, adapté aux besoins de l'époque. D'autres enfin, les plus nombreux, n'ont laissé pour toutes traces que des vestiges d'aménagement hydraulique, détruits par le feu ou simplement remplacés par un équipement contemporain.

As part of its thematic investigation of the Vosges mountain's small hydraulic industrial sites, the General Inventory service of the Lorraine region undertook a study of water-powered saw-mills. More than 250 sites are mentioned in the archives; 115 sites, from slight vestiges to complete saw-mills, were identified in the field and 25 were the subject of individual notices published on the Ministry's national heritage database, Mérimée. One was listed as a historic monuments, completing a corpus of sites already protected at an earlier date. Certain sites have been known for a long time, protected as historic monuments and exploited as tourist attractions. Others, even though their original machinery might remain, can be found in a state of complete abandon, or, less frequently, restored by private owners and not open to public visits. Still others have suffered from the multiple vicissitudes that affect industrial buildings, being constantly modified and adapted to the needs of the time. Of others, finally-the majority-there are only vestiges of the water-power organisation, the buildings having been destroyed by fire or simply replaced by contemporary equipment.

\section{INDEX}

Mots-clés : scierie, montagne vosgienne, haut-fer, hydraulique, turbine, sagard, Mandray, Xamontarupt, embiellage

Keywords : Saw-mill, hydropower, turbine, 'sagard' (saw-mill worker), Mandray, Xamontarupt, mechanical gearing

\section{AUTEUR}

\section{JEAN-YVES HENRY}

Chercheur au service de l'Inventaire général, région Alsace-Champagne-Ardenne-Lorraine jeanyves.henry@lorraine.eu 